\title{
A stellar census in globular clusters with MUSE: Binaries in NGC 3201^
}

\author{
Benjamin Giesers ${ }^{1}$, Sebastian Kamann ${ }^{2}$, Stefan Dreizler ${ }^{1}$, Tim-Oliver Husser ${ }^{1}$, Abbas Askar ${ }^{3}$, Fabian Göttgens ${ }^{1}$, \\ Jarle Brinchmann ${ }^{4,5}$, Marilyn Latour ${ }^{1}$, Peter M. Weilbacher ${ }^{6}$, Martin Wendt ${ }^{7}$, and Martin M. Roth ${ }^{6}$ \\ ${ }^{1}$ Institut für Astrophysik, Georg-August-Universität Göttingen, Friedrich-Hund-Platz 1, 37077 Göttingen, Germany \\ e-mail: giesers@astro.physik.uni-goettingen.de \\ 2 Astrophysics Research Institute, Liverpool John Moores University, 146 Brownlow Hill, Liverpool L3 5RF, UK \\ ${ }^{3}$ Lund Observatory, Department of Astronomy and Theoretical Physics, Lund University, Box 43, 22100 Lund, Sweden \\ ${ }^{4}$ Instituto de Astrofísica e Ciências do Espaço, Universidade do Porto, CAUP, Rua das Estrelas, 4150-762 Porto, Portugal \\ ${ }^{5}$ Leiden Observatory, Leiden University, PO Box 9513, 2300 RA Leiden, The Netherlands \\ 6 Leibniz-Institut für Astrophysik Potsdam (AIP), An der Sternwarte 16, 14482 Potsdam, Germany \\ 7 Institut für Physik und Astronomie, Universität Potsdam, Karl-Liebknecht-Str. 24/25, 14476 Golm, Germany
}

Received 28 June 2019 / Accepted 17 September 2019

\begin{abstract}
We utilise multi-epoch MUSE spectroscopy to study binary stars in the core of the Galactic globular cluster NGC 3201. Our sample consists of 3553 stars with 54883 spectra in total comprising 3200 main-sequence stars up to 4 magnitudes below the turn-off. Each star in our sample has between 3 and 63 (with a median of 14) reliable radial velocity measurements within five years of observations. We introduce a statistical method to determine the probability of a star showing radial velocity variations based on the whole inhomogeneous radial velocity sample. Using HST photometry and an advanced dynamical MOCCA simulation of this specific cluster we overcome observational biases that previous spectroscopic studies had to deal with. This allows us to infer a binary frequency in the MUSE field of view and enables us to deduce the underlying true binary frequency of $(6.75 \pm 0.72) \%$ in NGC 3201 . The comparison of the MUSE observations with the MOCCA simulation suggests a large portion of primordial binaries. We can also confirm a radial increase in the binary fraction towards the cluster centre due to mass segregation. We discovered that in the core of NGC 3201 at least $(57.5 \pm 7.9) \%$ of blue straggler stars are in a binary system. For the first time in a study of globular clusters, we were able to fit Keplerian orbits to a significant sample of 95 binaries. We present the binary system properties of eleven blue straggler stars and the connection to SX Phoenicis-type stars. We show evidence that two blue straggler formation scenarios, the mass transfer in binary (or triple) star systems and the coalescence due to binary-binary interactions, are present in our data. We also describe the binary and spectroscopic properties of four sub-subgiant (or red straggler) stars. Furthermore, we discovered two new black hole candidates with minimum masses $(M \sin i)$ of $(7.68 \pm 0.50) M_{\odot},(4.4 \pm 2.8) M_{\odot}$, and refine the minimum mass estimate on the already published black hole to $(4.53 \pm 0.21) M_{\odot}$. These black holes are consistent with an extensive black hole subsystem hosted by NGC 3201 .
\end{abstract}

Key words. binaries: general - blue stragglers - stars: black holes - techniques: radial velocities techniques: imaging spectroscopy - globular clusters: individual: NGC 3201

\section{Introduction}

Multi-star systems are not only responsible for an abundance of extraordinary objects like cataclysmic variables, millisecond pulsars, and low-mass X-ray binaries, but can also be regarded as a source of energy: Embedded in the environment of other stars, like in star clusters, the gravitational energy stored in a multi-star system can be transferred to other stars. In globular clusters with stellar encounters on short timescales this energy deposit is for example delaying the core collapse (Goodman \& Hut 1989). From a thermodynamic point of view, multi-star systems in globular clusters act as a heat source. The prerequisite for this mechanism is, of course, that globular clusters do contain multi-star systems at some point during their lifetime. For

* The full radial velocity sample (Table A.1) is only available at the CDS via anonymous ftp to cdsarc.u-strasbg. fr (130.79.128.5) or via http://cdsarc.u-strasbg.fr/viz-bin/cat/J/A+A/632/ A3 the main-sequence field stars $>50 \%$ (Duquennoy \& Mayor 1991; Duchêne \& Kraus 2013) in our Milky Way are in multi-star systems. In globular clusters, binary fractions are typically lower than in the field (Milone et al. 2012), but can reach values around $\sim 50 \%$ (e.g Sollima et al. 2007; Milone et al. 2012) in central regions. This radial gradient can be explained by mass segregation and indeed is reproduced in simulations (e.g. Fregeau et al. 2009; Hurley et al. 2007). The initial fraction of multi-star systems in globular clusters is changed due to dynamical formation and destruction processes caused by stellar encounters, as well as stellar evolution during the (typical long) life time of Galactic globular clusters. The primordial fraction of multi-star systems to single stars for globular clusters therefore cannot be easily inferred from observations (Hut et al. 1992). As a result, the initial fraction is poorly constrained and simulations use values in a wide range from 5\% (Hurley et al. 2007) to 100\% (Ivanova et al. 2005). Current observations are still consistent with this wide range in primordial binary fractions, depending on which orbit distribution is assumed (Leigh et al. 2015). 
Until today there is only one technique with a high discovery efficiency for multi-star systems, namely high precision photometry (Sollima et al. 2007; Milone et al. 2012). It uses the fact that binary stars with both components contributing to the total brightness of one unresolved source have a position in the colour-magnitude-diagram (CMD) differing from single stars. For example, a large number of binaries is located to the brighter (redder) side of the main-sequence (MS). This method is sensitive to binaries in the MS with arbitrary orbital period and inclination. However, no constraints on orbital parameters are possible. The advantage is that this method is efficient in terms of observing time and produces large statistical robust samples. The disadvantage is that in terms of individual binary system properties it only has access to the biased mass-ratio parameter. Milone et al. (2012) investigated 59 Galactic globular clusters with this method using the Hubble Space Telescope (HST). They studied MS binaries with a mass ratio $q>0.5$ and found in nearly all globular clusters a significantly smaller binary frequency than $50 \%$. The results show a higher concentration of binaries in the core with a general decreasing binary fraction from the centre to about two core radii by a factor of $\sim 2$. A significant anti-correlation between the cluster binary frequency and its absolute luminosity (mass) was found. Additionally, the authors confirm a significant correlation between the fraction of binaries and the fraction of blue straggler stars (BSS), indicating a relation between the BSS formation mechanism and binaries.

In the literature there are almost no spectroscopic studies of the binarity in globular clusters. One systematic search was done in the globular cluster M4 based on 5973 spectra of 2469 stars by Sommariva et al. (2009). They discovered 57 binary star candidates and derived a lower limit of the total binary fraction of $(3.0 \pm 0.3) \%$ for M4.

In light of the important role of binaries in globular clusters, we designed the observing strategy of the MUSE globular cluster survey (Kamann et al. 2018) such that we can infer the binary fraction of every cluster in our sample. For all clusters we aim for at least three epochs of each pointing. For a selection of clusters, namely NGC 104 (47 Tuc), NGC 3201, and NGC 5139 ( $\omega$ Cen), we are also able to study individual binary systems in more detail. This paper concentrates on the globular cluster NGC 3201. NGC 3201 has, apart NGC 5139, the largest half-light and core radius of all globular clusters in our survey. Its binary fraction within the core radius appears to be relatively high $((12.8 \pm 0.8) \%$, Milone et al. 2012). In Giesers et al. (2018), we reported a quiescent detached stellar-mass black hole with $4.36 \pm 0.41) M_{\odot}$ in this cluster, the first black hole found by a blind spectroscopic survey. Based upon our discovery, NGC 3201 was predicted to harbour a significant population of black holes (see Kremer et al. 2018; Askar et al. 2018a). In our MUSE data, NGC 3201 has the deepest observations, the most epochs to date, and the least crowding. It is therefore the ideal case for our purpose.

This paper is structured as follows. In Sect. 2 we describe peculiar objects which are important in the context of binary star studies in NGC 3201. In Sect. 3 the observations, data reduction and selection of our final sample are discussed. In Sect. 4 we present a MOCCA simulation of the globular cluster NGC 3201 that we used to create a mock observation for comparisons and verifying the following method. We introduce a statistical method to determine the variability of radial velocity measurements and use the method to determine the binary fraction of NGC 3201 in Sect. 5. In Sect. 6 we explain our usage of the tool THE JOKER to find orbital solutions to individual binary systems and present their orbital parameter distributions. We discuss peculiar objects in our data, such as blue straggler stars, sub-subgiants and black hole candidates in Sect. 7. We end with the conclusions and outlook in Sect. 8.

\section{Peculiar objects in globular clusters}

\subsection{Blue straggler stars}

In most globular clusters a collection of blue straggler stars can be easily found in the CMD along an extension of the MS beyond the turn-off point. Their positions indicate more massive and younger stars than those of the MS. Without continuing star formation due to lacking gas and dust in globular clusters, mass transfer from a companion is the usual explanation for the formation of a blue straggler. This mass transfer could be on long timescales, like in compact binary systems, or on short timescales, as for collisions of two stars (in a binary system or of a priori not associated stars). However, it is still unclear which of these processes contribute to the formation of blue stragglers and how often. Ferraro et al. (1997) discovered a bimodal radial distribution of blue stragglers in M3 and suggested that the blue stragglers in the inner cluster are formed by stellar collisions and those in the outer cluster from merging primordial binaries. Sollima et al. (2008) identified that the strongest correlation in low-density globular clusters is between the number of blue stragglers and the binary frequency of the cluster. They suggested that the primordial binary fraction is one of the most important factors for producing blue stragglers and that the collisional channel to form blue stragglers has a very small efficiency in low-density globular clusters.

Hypki \& Giersz (2017) performed numerical simulations of globular clusters with various initial conditions. They found that the number of evolutionary blue stragglers (formed in a compact binary system) is not affected by the density of the globular cluster. In contrast, the number of dynamically created blue stragglers (by collisions between stars) correlates with the density. The efficiencies of both channels strongly depend on the initial semi-major axes distributions. Wider initial orbits lead to more dynamically created blue stragglers. Finally, they found in all globular cluster models at the end of the simulation a constant ratio between the number of blue stragglers in binaries and as single stars $R_{\mathrm{B} / \mathrm{S}} \sim 0.4$.

A mass distribution of blue stragglers has been estimated by Fiorentino et al. (2014) from pulsation properties of blue stragglers in the globular cluster NGC 6541. They found a mass of $(1.06 \pm 0.09) M_{\odot}$, which is significantly in excess of the cluster MS turn-off mass $\left(0.75 M_{\odot}\right)$. Baldwin et al. (2016) found an average mass of blue straggler stars of $(1.22 \pm 0.12) M_{\odot}$ in 19 globular clusters. Geller \& Mathieu (2011) found a surprisingly narrow blue straggler companion mass distribution with a mean of $0.53 M_{\odot}$ in long-period binaries in the open cluster NGC 188. They suggested that this conclusively rules out a collisional origin in this cluster, as the collision hypothesis predicts significantly higher companion masses in their model.

\subsection{Sub-subgiant stars}

A star in a cluster is called a sub-subgiant (SSG) if its position in the CMD is redder than MS turn-off stars (and its binaries) and fainter than normal subgiant stars. These stars are related to the so called red stragglers (RS) which are redder than normal (red) giant stars. For our study this distinction is not important and we call them hereafter sub-subgiants. The evolution of these stars cannot be explained by single star evolution. 
Geller et al. (2017a) describe in detail the demographics of SSGs in open and globular clusters and conclude that half of their SSG sample are radial velocity binary stars with typical periods less than $15 \mathrm{~d}, 58 \%$ are $\mathrm{X}$-ray sources, and $33 \%$ are $\mathrm{H} \alpha$ emitters. The formation scenarios explained in Leiner et al. (2017) for SSG and RS are isolated binary evolution, the rapid stripping of a subgiant's envelope, or stellar collisions. So far, from observations and simulations, no channel can be excluded, but it seems that the binary channel is dominant in globular clusters (Geller et al. 2017b).

\subsection{SX Phoenicis-type stars}

SX Phoenicis-type (SXP) stars are low-luminosity pulsators in the classical instability strip with short periods. In globular clusters they appear as blue straggler stars with an enhanced helium content (Cohen \& Sarajedini 2012). SXP stars show a strong period-luminosity relation, from which distances can be measured. The period range is from $0.02 \mathrm{~d}$ to $0.4 \mathrm{~d}$ with typical periods around $\sim 0.04 \mathrm{~d} \approx 1 \mathrm{~h}$. SXP stars are thought to be formed in binary evolution, but it is not clear whether they are indicative of a particular blue straggler formation channel. The prototype star SX Phoenicis shows a radial velocity amplitude of $19 \mathrm{~km} \mathrm{~s}^{-1}$ due to radial pulsation modes (Kim et al. 1993).

\subsection{Black holes}

Observers are searching for two kinds of black holes in globular clusters. On the one hand, we have no conclusive evidence for central intermediate-mass black holes (IMBH, see Baumgardt 2017; Tremou et al. 2018). One of the challenges in searching for IMBHs is that typical signatures, such as a central cusp in the surface brightness profile or the velocity dispersion profile can also be explained by a binary star or stellar-mass black hole population (for Omega Cen, see Zocchi et al. 2019). On the other hand, there are known stellar-mass black holes in Galactic globular clusters (e.g. Strader et al. 2012; Giesers et al. 2018). If they are retained, stellar-mass black holes should quickly migrate to the cluster cores due to mass segregation, and strongly affect the evolution of their host globular clusters (e.g. Kremer et al. 2018; Arca Sedda et al. 2018; Askar et al. 2018a, 2019). However, the retention fraction of black holes in globular clusters is highly unknown, which also limits our abilities to interpret gravitational wave detections. For NGC 3201, Askar et al. (2018a) and Kremer et al. (2018) predicted that between 100 and more than 200 stellar-mass black holes should still be present in the cluster, with up to ten in a binary system with a MS star.

\section{Observations and data reduction}

The observational challenge in globular clusters is the crowded field resulting in multiple star blends. For photometric measurements of dense globular clusters, instruments such as the ones attached to the HST have just sufficient spatial resolution to get independent measurements of stars down the MS. In the past, spectroscopic measurements of individual globular cluster stars (with a reasonable spectroscopic resolution) were limited by crowding in the cluster centre. Since 2014 we are observing 27 Galactic globular clusters (PI: S. Kamann, formerly S. Dreizler) with the integral field spectrograph Multi Unit Spectroscopic Explorer (MUSE) at the Very Large Telescope (VLT; Kamann et al. 2018). In the wide field mode, MUSE covers a $1^{\prime} \times 1^{\prime}$ field of view (FoV) with a spatial sampling of $0.2^{\prime \prime}$ and a

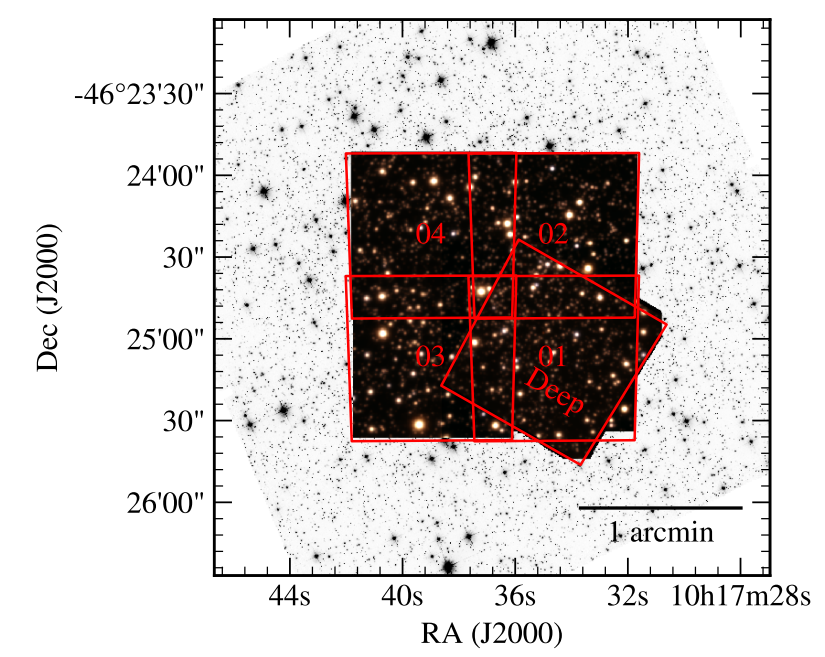

Fig. 1. Pointing chart of the MUSE instrument for the globular cluster NGC 3201. The inverted background image is from the ACS globular cluster survey (Sarajedini et al. 2007). The MUSE FoV is a collapsed RGB image created from the spectral cubes. Note the overlapping areas between the pointings.

spectral sampling of $1.25 \AA$ (resolving power of $1770<R<$ 3590 ) in the wavelength range from 4750 to $9350 \AA$. MUSE allows us to extract spectra of some thousand stars per exposure.

The overall aim of our stellar census in globular clusters with MUSE is twofold. On the one hand we investigate the spectroscopic properties of more than half a million of cluster members down to the MS. On the other hand we investigate the dynamical properties of globular clusters. Upcoming publications are in the field of multiple populations (Husser et al. 2019; Latour et al. 2019; Kamann et al., in prep.), the search for emission line objects (Göttgens et al. 2019a,b), cluster dynamics like the overall rotation (Kamann et al. 2018), and gas clouds in direction of the clusters (Wendt et al. 2017). Since this survey represents the first blind spectroscopic survey of globular cluster cores, we expect many unforeseen discoveries.

For this paper, we used all observations of NGC 3201 obtained before May 2019. The pointing scheme can be seen in Fig. 1. In addition to the pointings listed in Kamann et al. (2018), we also implemented a deep field mainly to go deeper on the MS. Table 1 lists the number of visits and total integration times for the different pointings. Our data include adaptive optics (AO) observations, our standard observing mode since the AO system was commissioned in October 2017. The AO observations are treated in the same way as our previous observations. The only difference is that a wavelength window of each spectrum around the sodium lines ( $5805 \AA-5965 \AA$ ) has to be masked due to the AO laser emission. During each visit, the pointing was observed with three different instrument derotator angles $(0,90$, $180^{1}$ degrees) in order to reduce systematic effects of the individual MUSE spectrographs.

Figure 1 shows our observation scheme for NGC 3201. We secured at least 12 observations per pointing (see Table 1 and Fig. 2). Thanks to the overlapping pointings, up to 63 independent observations per star are available in the central region (see Fig. 3).

To differentiate between radial velocity signals from short period binaries and those of longer period binaries, we observed each of the four pointings twice with a separation of two to three

1 The deep pointing was observed with an additional derotator angle of 270 degrees. 
Table 1. Exposures, observations, and integration time per pointing.

\begin{tabular}{cccc}
\hline \hline Pointing & Exposures & Observations & Total time [min.] \\
\hline 01 & 49 & 17 & 163 \\
02 & 49 & 15 & 143 \\
03 & 44 & 15 & 143 \\
04 & 36 & 12 & 114 \\
Deep & 16 & 4 & 160 \\
\hline$\sum$ & 194 & 63 & 723 \\
\hline
\end{tabular}

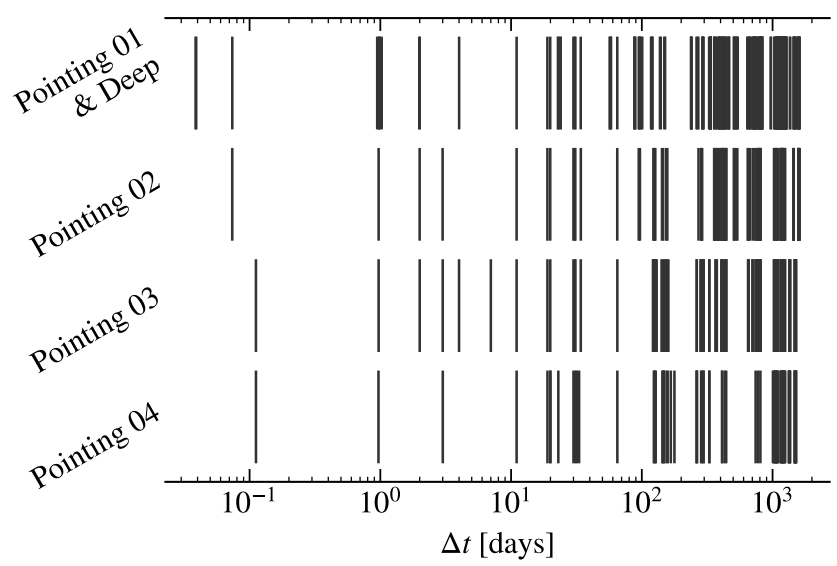

Fig. 2. All possible measurement baselines per star and pointing. $\Delta t$ is the time between two observations.

hours in a single night. We already had this distinction sometimes for the stars observed within the same night in the overlapping region of two pointings (like for the published black hole candidate). Without that short baseline a hard binary with a period below one day could mimic the signal of a companion of a black hole candidate at a longer period (temporal aliasing).

The reduction of all exposures was carried out using the standard MUSE pipeline (Weilbacher et al. 2012, 2014). The extraction from the final product of each visit (epoch) is done by a PSF-fitting technique implemented in the software PAMPELMUSE (Kamann et al. 2013). After an extraction is finished the individual spectra are fitted against a synthetic stellar library to determine stellar properties with SPEXXY (Husser et al. 2013). The extraction and stellar parameter fitting methods are summarised in Husser et al. (2016) and Kamann et al. (2018).

\subsection{Radial velocities}

In this study, we want to investigate if a given star is in a binary system using the radial velocity method. Binaries that are able to survive in the dense environment of a globular cluster are so tight, that even HST is not able to spatially resolve the binary components at the distance of NGC 3201. For this reason, the binary system will appear as a single point-like source. Except for the case when both stars have similar brightness (see Sect. 4 on how we handle these binaries), the extracted MUSE spectrum will be dominated by one star. The measured radial velocity variation of this star can be used to determine the properties of the binary system indirectly.

Since we aim for the highest possible radial velocity precision, we checked if the standard wavelength calibration of the MUSE instrument and the uncertainties calculated by the extraction and fitting routines are reliable. For this purpose we analysed the wavelength shift of the telluric lines in the extracted

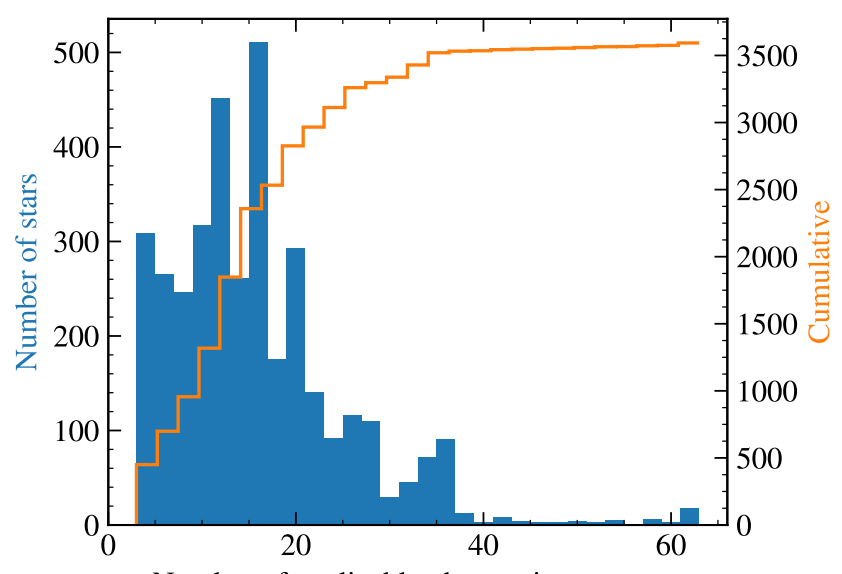

Number of applicable observations per star

Fig. 3. Histogram of applicable (accepted after filtering) number of observations per star (blue) and cumulative distribution of the same data (orange).

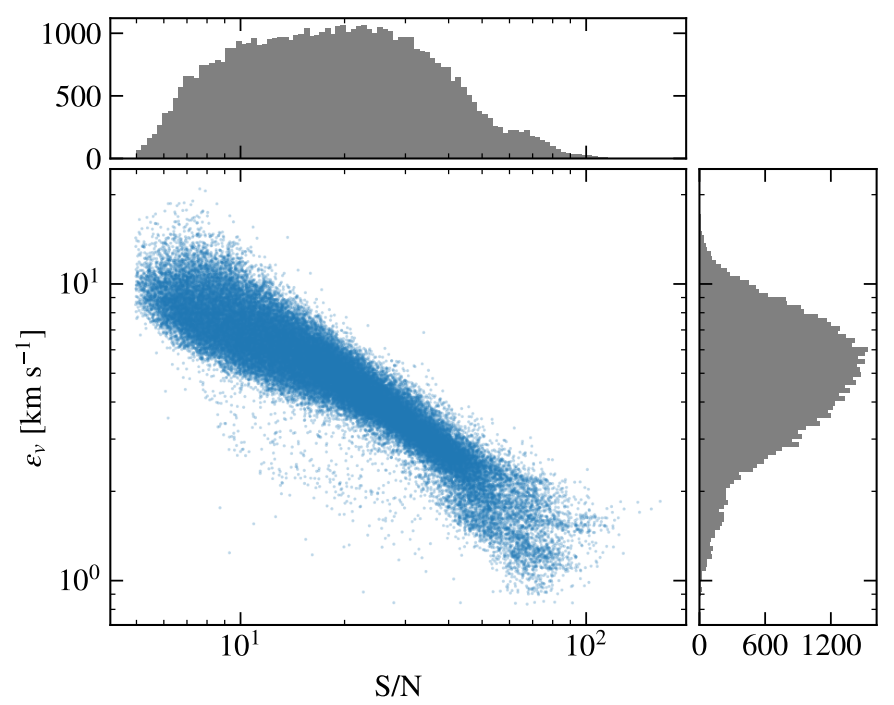

Fig. 4. Radial velocity uncertainties $\epsilon_{v}$ of all spectra in the sample used for NGC 3201 as a function of the spectral signal-to-noise.

spectra per observation to correct for tiny deviations in the wavelength solution of the pipeline (see Sect. 4.2 in Kamann et al. 2018 , for details). We also cross checked the radial velocity of every spectrum obtained by the full-spectrum fit using a crosscorrelation of the spectrum with a PHOENIX-library template (Husser et al. 2013) (see criterion in Sect. 3.2). Figure 4 shows the resulting uncertainties $\epsilon_{v}$ as a function of $S / N$ of the spectra in our final sample of NGC 3201. We get reliable radial velocities from $S / N>5$ spectra (Kamann et al. 2018). At $S / N=10$ the uncertainties are $\epsilon_{v} \approx(7.0 \pm 1.7) \mathrm{km} \mathrm{s}^{-1}$, at $S / N \gtrsim 50$ the uncertainties are $\epsilon_{v} \approx(1.7 \pm 0.5) \mathrm{km} \mathrm{s}^{-1}$. These uncertainties represent our spectroscopic radial velocity precision, the accuracy of the MUSE instrument (wavelength shift between two observations) is below $1 \mathrm{~km} \mathrm{~s}^{-1}$ (Kamann et al. 2018).

\subsection{Selection of the final sample}

For the sample selection two things are crucial. Firstly, we only want reliable radial velocities with Gaussian distributed uncertainties. Secondly, our sample should only consist of single and multiple star systems which are cluster members, excluding 
Galactic field stars. Finally, stars that mimic radial velocity variations due to intrinsic pulsations should be excluded. To ensure this the following filters are applied:

- The reliability of every single spectrum is ensured by the subsequent criteria, which are either 0 (false) and 1 (true). We apply a cut for the signal to noise $S / N$ of a spectrum

$R_{S / N}=S / N \geq 5$.

The quality of the cross-correlation is checked using the $r$-statistics and FWHM defined by Tonry \& Davis (1979)

$R_{\mathrm{cc}}=r \geq 4 \wedge F W H M>10 \AA$.

A plausible uncertainty $\epsilon_{\mathrm{v}, \mathrm{cc}}$ of the cross-correlation radial velocity $v_{\mathrm{cc}}$

$R_{\epsilon_{\mathrm{v}, \mathrm{cc}}}=\epsilon_{\mathrm{v}, \mathrm{cc}}>0.1 \mathrm{~km} \mathrm{~s}^{-1}$,

and a plausible uncertainty $\epsilon_{\mathrm{V}}$ of the full-spectrum fit radial velocity $v$

$R_{\epsilon_{\mathrm{v}}}=\epsilon_{\mathrm{v}}>0.1 \mathrm{~km} \mathrm{~s}^{-1}$

is ensured. We check that the velocity is within $3 \sigma$ of the cluster velocity $v_{\text {cluster }}$ and the cluster dispersion $\sigma_{\mathrm{v}, \text { cluster }}$ including the standard deviation taken from the Harris (1996, 2010 edition) catalogue. For typical binary amplitudes we add $30 \mathrm{~km} \mathrm{~s}^{-1}$ to the tolerance, yielding

$R_{v}=\frac{\left|v-v_{\text {cluster }}\right|}{\sqrt{\epsilon_{\mathrm{v}}^{2}+\sigma_{\mathrm{v}, \text { cluster }}^{2}+\left(30 \mathrm{~km} \mathrm{~s}^{-1}\right)^{2}}} \leq 3$.

The last criterion is the check if cross-correlation and fullspectrum fit are compatible within $3 \sigma$ with each other,

$R_{v=v_{\mathrm{cc}}}=\frac{\left|v-v_{\mathrm{cc}}\right|}{\sqrt{\epsilon_{\mathrm{v}}^{2}+\epsilon_{\mathrm{v}, \mathrm{cc}}^{2}}} \leq 3$.

We combine all these criteria in an empirically weighted equation, which gives us a result between 0 and 1 ,

$R_{\text {total }}=\left(2 R_{S / N}+10 R_{\mathrm{cc}}+R_{\epsilon_{\mathrm{v}, \mathrm{cc}}}+3 R_{v}+2 R_{\epsilon_{\mathrm{v}}}+5 R_{v=v_{\mathrm{cc}}}\right) / 23$.

A radial velocity is considered as reliable if the reliability $R_{\text {total }}$ surpasses $80 \%$.

- We exclude spectra which are extracted within 5 px from the edge of a MUSE cube to avoid systematic effects.

- We exclude spectra where PAMPELMUSE was unable to deblend multiple nearby HST/ACS sources (this is of course seeing dependent and affects mostly faint sources).

- To identify confusion or extraction problems in a MUSE spectrum, we calculated broad-band magnitudes from the spectrum in the same passband that was used in the extraction process and calculated the differences between input and recovered magnitudes. We accept only spectra with a magnitude accuracy $>80 \%$ (see Sect. 4.4 in Kamann et al. 2018, for more details).

- In some low $S / N$ cases the cross-correlation and fullspectrum fit returned similar but wrong radial velocities due to noise in the spectrum. These could be outliers by several $100 \mathrm{~km} \mathrm{~s}^{-1}$ introducing a strong variation in the signal and passing the reliability criteria described before. To find outliers we generally compare all radial velocities $v_{i}$ (with their uncertainties $\left.\epsilon_{v, i}\right)$ of a single star with each other. We define the set of all radial velocities as $A=\left\{v_{i}\right\}$. An outlier $v_{x}$ in the subset $B=A \backslash\left\{v_{x}\right\}$ of all radial velocities except the inspected radial velocity, is identified with

$\frac{\sigma_{A}}{\sigma_{B}}>\kappa \wedge \frac{\left|v_{x}-\tilde{B}\right|}{\epsilon_{v, x}+\sigma_{B}}>\kappa \quad$ with $\kappa=3$.

$\tilde{B}$ is the median of the radial velocities $v_{i}$ in the set $B . \sigma$ is the standard deviation of the corresponding quantity. Using this equation outliers are identified and excluded from further analyses.

- We manually set the binary probability in our sample to 0 for 10 RR Lyrae- and 5 SX Phoenicis-type stars known in the Catalogue of Variable Stars in Galactic Globular Clusters (Clement et al. 2001; Clement 2017) and Arellano Ferro et al. $(2014)^{2}$.

- Since NGC 3201 has a heliocentric radial velocity of $(494.0 \pm 0.2) \mathrm{km} \mathrm{s}^{-1}$ and a central velocity dispersion of $(5.0 \pm$ $0.2) \mathrm{km} \mathrm{s}^{-1}$ (Harris 1996, 2010 edition), we select members by choosing stars with mean radial velocities $>400 \mathrm{~km} \mathrm{~s}^{-1}$.

- Our final sample only consists of member stars with at least 3 spectra per star after all filters have been applied.

We end up with 3553 stars and 54883 spectra out of 4517 stars and 68084 spectra in total. Due to different observational conditions the stars near the confusion limit cannot be extracted reliably from every observation. Figure 3 shows a histogram of all applicable observations per star for the final sample of NGC 3201. We have 3285 (91\%) stars with five or more epochs, $2637(73 \%)$ with ten or more epochs, and 918 (26\%) stars with 20 or more epochs.

\subsection{Mass estimation}

We estimate the mass of each star photometrically by comparing its colour and magnitude from the ACS globular cluster survey (Sarajedini et al. 2007; Anderson et al. 2008) with a PARSEC isochrone (Bressan et al. 2012). For the globular cluster NGC 3201, we found the best matching isochrone compared to the whole ACS CMD with the isochrone parameters $[\mathrm{M} / \mathrm{H}]=-1.39$ dex (slightly above the comparable literature value $[\mathrm{Fe} / \mathrm{H}]=-1.59$ dex, Harris 1996, 2010 edition), age $=11 \mathrm{Gyr}$, extinction $E_{B-V}=0.26$, and distance $=4.8 \mathrm{kpc}$. The mass for each star is determined from its nearest neighbour on the isochrone. For blue straggler stars we assume a mass of $(1.20 \pm 0.05) M_{\odot}$ (Baldwin et al. 2016) instead.

\subsection{The electronic catalogue}

An outline of the final sample of the radial velocities is listed in Table A.1. The columns ACS Id, position (RA, Dec), and $\mathrm{V}$ equivalent magnitude from the ACS catalogue (Sarajedini et al. 2007) are followed by the mean observation date of the combined exposures (BMJD), the barycentric corrected radial velocity $v_{\mathrm{r}}$, and its uncertainty $\epsilon_{v}$ from our MUSE observations. The last column contains the probability for variability in radial velocity $P\left(\chi_{i}^{2}, v_{i}\right)$, determined as described in Sect. 5 . Three versions of the catalogue, one with the full final sample (see Sect. 3.2), one with non-member stars, and one with the

2 The RR Lyrae-type stars are the brightest stars showing radial velocity variations in our sample and we verified that some of them show Keplerian-like signals with periods $<1 \mathrm{~d}$ and high eccentricities. 
Table 2. Globular cluster properties for the MOCCA model in comparison to observed values.

\begin{tabular}{lll}
\hline \hline RA & $10^{\mathrm{h}} 17^{\mathrm{m}} 36.82^{(a)}$ \\
Dec & $-46^{\circ} 24^{\prime} 44^{\prime \prime} 9^{(a)}$ \\
Distance to Sun & $4.9 \mathrm{kpc}^{(a)}$ \\
Metallicity [Fe/H] & $-1.59 \mathrm{dex}$ \\
Barycentric radial velocity & $(494.0 \pm 0.2) \mathrm{km} \mathrm{s}^{-1(a)}$ \\
\hline & MOCCA & Literature \\
\hline Central velocity dispersion $\left[\mathrm{km} \mathrm{s}^{-1}\right]$ & $5.23^{(*)}$ & $(5.0 \pm 0.2)^{(a)}$ \\
Core radius [pc] & 1.54 & $1.85^{(a)}$ \\
Half-light radius [pc] & 4.16 & $4.42^{(a)}$ \\
Total $V$-band luminosity $\left[L_{\odot}\right]$ & $9.27 \times 10^{4}$ & $8.17 \times 10^{4(a)}$ \\
Central surface brightness $\left[L_{\odot} / \mathrm{pc}^{2}\right]$ & $2.25 \times 10^{3}$ & $9.14 \times 10^{2}(a)$ \\
Age [Gyr] & 12 & $(12.0 \pm 0.8)^{(b)}$ \\
Mass $\left[M_{\odot}\right]$ & $2.3 \times 10^{5}$ & $(1.49 \pm 0.09) \times 10^{5(c)}$ \\
Total binary fraction [\%] & 8.72 & - \\
\hline
\end{tabular}

Notes. ${ }^{(*)}$ Computed for stars brighter than 3 mags below MS turn-off. References. ${ }^{(a)}$ Harris $\left(1996,2010\right.$ edition) catalogue; ${ }^{(b)}$ Dotter et al. (2010); ${ }^{(c)}$ Baumgardt \& Hilker (2018).

RR Lyrae and SX Phoenicis stars are available at the CDS and on the project website ${ }^{3}$.

\section{MOCCA simulation of NGC 3201}

We want to compare our observations of binaries in NGC 3201 with models. Our first attempt using a toy model with realistic binary parameter distributions for this specific cluster like of period, eccentricity, mass ratio, etc. failed since the creation of binaries from these distributions is unrealistic. Moe \& Di Stefano (2017) demonstrated that randomly drawing values of these orbital parameter distributions does not lead to realistic currently observed binary distributions even when the parameter distributions themselves are realistic. Especially not for globular clusters where dynamical interactions between stars play an important role. Thus we have to use more sophisticated simulations of globular clusters including all relevant physics for the cluster dynamics and the binary evolution. Simulating the dynamical evolution of realistic globular clusters in a Galactic potential up to a Hubble time is computationally expensive.

\subsection{The model}

An approach that has been used to extensively simulate realistic globular cluster models is the Monte Carlo method for stellar dynamics that was first developed by Hénon (1971). This method combines the statistical treatment of relaxation with the particle based approach of direct $N$-body methods to simulate the long term evolution of spherically symmetric star clusters. The particle approach of this method enables the implementation of additional physical processes (including stellar and binary evolution) and is much faster than direct $N$-body methods. In recent years, an extensive number of globular cluster models have been simulated with the MOCCA (MOnte Carlo Cluster simulAtor; Giersz 1998; Hypki \& Giersz 2013; Giersz et al. 2013; Askar et al. 2017; Hong et al. 2018; Belloni et al. 2019) and CMC (Cluster Monte Carlo) codes (Joshi et al. 2000; Pattabiraman et al. 2013; Morscher et al. 2015; Rodriguez et al. 2016; Chatterjee et al. 2017).

For the purpose of this project we needed a simulated globular cluster model that would have present-day observational properties comparable to NGC 3201 and that would also contain

\footnotetext{
https://musegc.uni-goettingen.de/
}

stellar-mass black holes like the one we found (Giesers et al. 2018). Therefore, we used results from the MOCCA Survey Database I (Askar et al. 2017) to identify initial conditions for a model that had global properties close to NGC 3201 at an age of 12 Gyr. This model has been subsequently refined with additional MOCCA simulations based on slightly modified initial conditions to find an even better agreement with the properties of NGC 3201 at an age of 12 Gyr including total luminosity, core and half-light radii, central surface brightness, velocity dispersion and binary fraction.

The MOCCA code utilizes the Monte Carlo method to compute relaxation. Additionally, it uses the fewbody code (Fregeau et al. 2004, small number N-body integrator) to compute the outcome of strong interactions involving binary stars. MOCCA also makes use of the SSE (Hurley et al. 2000) and BSE (Hurley et al. 2002) codes to carry out stellar and binary evolution of all stars in the cluster. MOCCA results have been extensively compared with results from direct $N$-body simulations and they show good agreement for both the evolution of global properties and number of specific objects (Giersz et al. 2013; Wang et al. 2016).

The following initial parameters were used for the MOCCA model we simulated specifically for this work to have a comparison to NGC 3201: The initial number of objects was set to $N=5.5 \times 10^{5}$. The initial binary fraction was set to $50 \%$, resulting in $2.25 \times 10^{5}$ single stars and $5.5 \times 10^{5}$ stars in binary systems, thus in total $8.25 \times 10^{5}$ stars in the initial model. The initial binary properties were set as described in Belloni et al. (2017). The metallicity of the model was set to $\left(Z=5.1 \times 10^{-4}\right)$ to correspond to the observed metallicity of NGC 3201 provided in the Harris (1996, 2010 edition) catalogue. We used a King (1966) model with a central concentration parameter $\left(W_{0}\right)$ of 6.5 . The model had an initial half-mass radius of $1.65 \mathrm{pc}$ and a tidal radius of $165 \mathrm{pc}$. The initial stellar masses were sampled using a two-component Kroupa (2001) initial mass function (IMF) with masses in the range $0.08 M_{\odot}$ to $100 M_{\odot}$. Neutron star natal kicks were drawn from the Hobbs et al. (2005) distribution. For black holes, natal kicks were modified according to black hole masses that were computed following Belczynski et al. (2002). The properties of the evolved simulated model at $12 \mathrm{Gyr}$ are listed in Table 2 and compared to literature values. The MOCCA model for NGC 3201 is able to reproduce well the observational core radius, half-light radius, central velocity dispersion, and the $V$-band luminosity provided in Harris (1996, 2010 edition). The central surface brightness for the MOCCA model is a factor of 2.5 larger than the observed one which is unfortunately not given with an uncertainty. In contrast, McLaughlin \& van der Marel (2005), for example, found about twice the observational central surface brightness listed in Harris (1996, 2010 edition). Given the observational uncertainties and limitations, the agreement between the simulated model and the observed values are reasonable.

\subsection{The mock observation}

We project the simulation snapshot at $12 \mathrm{Gyr}$ in Cartesian coordinates using the COCOA code (Askar et al. 2018b). We use the distance modulus of NGC 3201 at a distance of $4.9 \mathrm{kpc}$ (Harris 1996, 2010 edition) to get the apparent magnitude of all stars. For binary stars we combine the two components into a single magnitude. We select the stars within the MUSE FoV (of all pointings). Additionally we use our observational completeness function for NGC 3201 (see Fig. 5) to select stars accordingly.

After we identified a set of stars comparable to the MUSE observations, we created mock radial velocities for these stars. To this aim, we let our observational data guide the simulation. For 


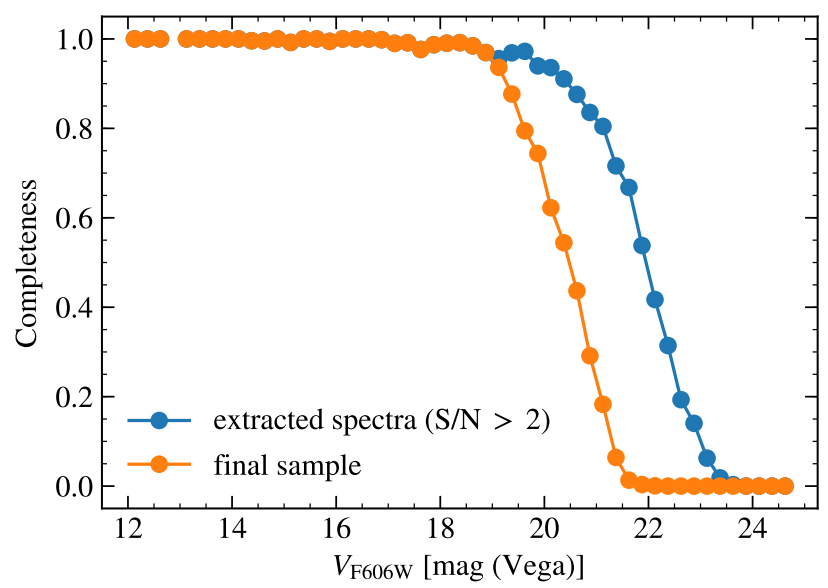

Fig. 5. Completeness as a function of magnitude for all MUSE observations of NGC 3201, using photometry from the ACS globular cluster survey (Sarajedini et al. 2007). The fraction of all possible extracted spectra to observed spectra within the MUSE FoV is shown. Blue: extracted spectra with $S / N>2$. Orange: extracted spectra in the final sample (see Sect. 3.2). (The uncertainties are smaller than the points.)

each observed star (in our MUSE sample) we randomly picked a MOCCA star with comparable magnitude. We then used the epochs of the observed star to create new radial velocity measurements. For single stars we simply assumed a radial velocity of $0 \mathrm{~km} \mathrm{~s}^{-1}$ for every time step. For binaries we calculated the radial velocity for the brighter component from a Keplerian orbit using the MOCCA properties of the binary system (masses, period, inclination, eccentricity, argument of periastron, and periastron time). However, due to the spectral resolution of MUSE its measured radial velocity amplitude will be influenced by the flux ratio of the two components of the binary system. From Monte Carlo simulations we found that the theoretical expected radial velocities $v_{\mathrm{r} \text {,theoretical }}$ are linearly damped with the flux ratio:

$v_{\mathrm{r}, \text { measured }}=v_{\mathrm{r}, \text { barycentre }}+\left(1-\frac{f_{2}}{f_{1}}\right) v_{\mathrm{r}, \text { theoretical }}$.

With the flux of the brighter component $f_{1}$ and the fainter component $f_{2} \cdot v_{\mathrm{r} \text {,barycentre }}$ is the radial velocity of the barycentre, which can be neglected if only relative velocities are of interest. In the case of both components having the same flux, the measured radial velocity $v_{\mathrm{r} \text {,measured }}$ amplitude will be zero. Thus, for example, twin MS binary stars are hard to find. In the case when the fluxes of the components are very different, the observed radial velocity amplitude corresponds to the amplitude of the brighter component. We apply this damping for every simulated radial velocity. Finally we scatter these radial velocities within the uncertainties taken from the MUSE corresponding measurements.

\section{The binary fraction of NGC 3201}

Section 4 reflects the efforts required to create realistic models for globular clusters. We therefore conceived a model independent approach to derive the binary frequency of a globular cluster, as well as the probability of an individual star to be radial velocity variable.

\subsection{A new method to detect variable stars}

We here introduce a general statistical method which could be applied to any inhomogeneous sample with a varying number of (a few) measurements and a large range of uncertainties. Here it is applied to radial velocity measurements: To detect radial velocity variations in a given star with $m$ measurements, we compute the $\chi_{i}^{2}$ for the set of measurements $x_{j}$ with uncertainties $\sigma_{j}$ to determine how compatible they are with the constant weighted mean $\bar{x}$ (null hypothesis) of the measurements:

$\chi_{i}^{2}=\sum_{j=1}^{m} \frac{1}{\sigma_{j}^{2}}\left(x_{j}-\bar{x}\right)^{2} \quad$ with $\quad \bar{x}=\frac{\sum_{j=1}^{m} \frac{x_{j}}{\sigma_{j}^{2}}}{\sum_{j=1}^{m} \frac{1}{\sigma_{j}^{2}}}$.

A star that shows radial velocity variations higher than the associated uncertainties will have reduced $\chi^{2}>1$ on average. In contrast, a star without significant variations will have reduced $\chi^{2} \approx 1$. As described in Sect. 3 each star in our sample has its own number of observations and therefore its own number of degrees of freedom ( $v=m-1$, see Fig. 3). Assuming Gaussian distributed uncertainties for all measurements and a common degree of freedom for all stars, we know the expected $\chi^{2}$-distribution in form of the cumulative distribution function (CDF) $F$ for the null hypothesis that all stars show constant signals. If there are binary stars with radial velocity variations in our sample, there will be an excess of higher $\chi^{2}$ values compared to the null hypothesis. Therefore, the empirical CDF computed from the measured $\chi^{2}$ will increase slower than the CDF computed using the null hypothesis.

For a chosen degree of freedom (e.g. $v=3$ ) we calculate the probability $\bar{P}$ of each star to match the null hypothesis through the comparison of the observed empirical distribution function (EDF) $F_{\text {observed }}$ with the expected CDF (comparable to the top panel in Fig. 6):

$\bar{P}\left(\chi_{i}^{2}, v_{i}=3\right)=\frac{1-F\left(\chi_{i}^{2}, 3\right)_{\text {theoretical }}}{1-F\left(\chi_{i}^{2}, 3\right)_{\text {observed }}}$.

Since the EDF $F_{\text {observed }}$ is simply the fraction of stars below a given $\chi^{2}$, we divide the number of stars we measured below a given $\chi^{2}$ with the expected number from the known CDF to calculate $P$ (comparable to the middle panel in Fig. 6). The probability of a star to be variable for a given degree of freedom $v$ is $P=1-\bar{P}$ :

$P\left(\chi_{i}^{2}, v_{i}\right)=\frac{F\left(\chi_{i}^{2}, v_{i}\right)_{\text {theoretical }}-F\left(\chi_{i}^{2}, v_{i}\right)_{\text {observed }}}{1-F\left(\chi_{i}^{2}, v_{i}\right)_{\text {observed }}}$.

To use the statistical power of the whole sample with the total number of stars $n$, this equation can be generalised to multiple degrees of freedom by noticing that $n F_{\text {theoretical }}$ is the expected number of stars below a given $\chi^{2}$. The (expected or observed) total number of stars below a given $\chi^{2}$, taking all available degrees of freedom into account, can be expressed using the number of the stars per degree of freedom $n_{v}$ and adding up the contributions from all degrees of freedom in a "super CDF":

$\widehat{S}\left(\chi_{i}^{2}\right)=\frac{1}{n} \sum_{v} F\left(\chi_{i}^{2}, v\right) n_{v}$,

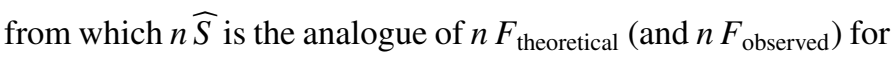
multiple degrees of freedom. Inserting this into Eq. (4) yields

$P\left(\chi_{i}^{2}, v_{i}\right)=\frac{n \widehat{S}\left(\chi_{k}^{2}\right)-k}{n-k} \quad$ with $k=\left|\left\{\chi^{2} \mid \chi^{2} / v<\chi_{i}^{2} / v_{i}\right\}\right|$

where $k$ is the number of stars with a reduced $\chi^{2}$ lower than the one of the given star, i.e. $\chi_{i}^{2} / v_{i}$ and $\chi_{k}^{2}$. 

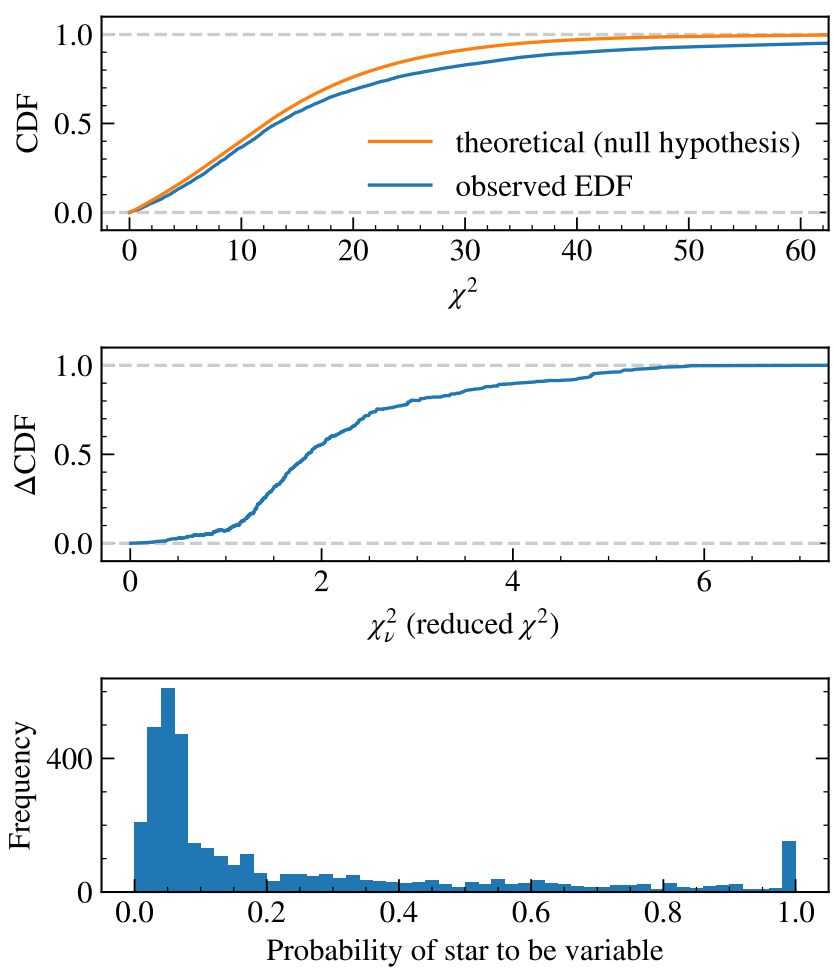

Fig. 6. Top panel: superposition of observed empirical distribution function (EDF) and expected theoretical cumulative distribution function (CDF) using the null hypothesis that no variable star exist in sample. Middle panel: resulting $\triangle \mathrm{CDF}$. Bottom panel: histogram of variability probabilities in the sample.

The upper panel of Fig. 6 shows the observed EDF and the theoretical "super CDF" $\widehat{S}$. Both functions deviate for increasing $\chi^{2}$, which indicates that stars in the sample not only show statistical variations. The middle panel shows the resulting $\triangle \mathrm{CDF}$ (Eq. (6)) for our sample. If we evaluate it with the reduced $\chi_{v}^{2}$ we get the probability for each star to vary in radial velocity. The bottom panel of Fig. 6 shows the resulting binary probability distribution of all NGC 3201 stars in our sample.

Comparing the number of stars with $P>0.5$ to the total number of stars yields the discovery binary fraction. We checked this approach using Monte Carlo simulations for different star samples of single and binary stars. It should be noted that at this $50 \%$ threshold there is a balance of false-positives and false-negatives, but that ensures a robust measurement for the overall statistics. Of course, for individual stars the acceptance probability to be a binary should be higher.

The statistical uncertainty of the binary fraction is calculated by the quadratic propagation of the uncertainty determined by bootstrapping (random sampling with replacement) the sample and the difference of the fraction for $P>0.45$ and $P>0.55$ divided by 2 as a proxy for the discriminability uncertainty between binary and single stars.

\subsection{Verification of the method on the MOCCA mock observation}

To verify the validity of this statistical method, we applied it to the MOCCA mock observation introduced in Sect. 4. For each star we calculated the variability probability according to Eq. (6). We also calculated a kind of semi-amplitude by bisection of the peak to peak of the simulated radial velocities $\left(\Delta v_{r} / 2\right)$ of each
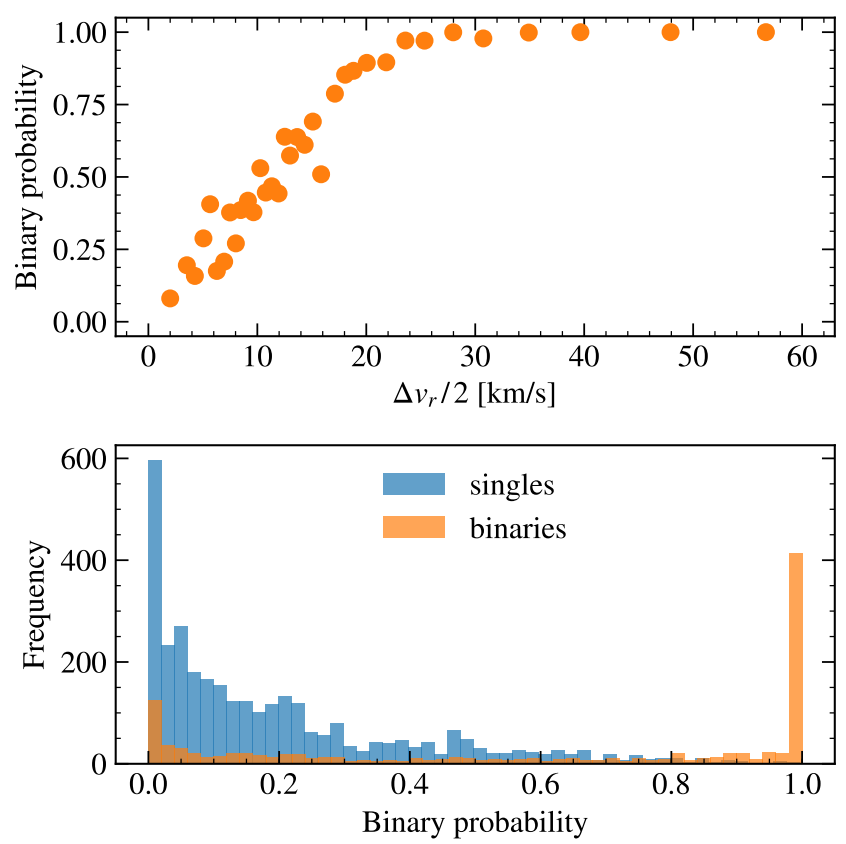

Fig. 7. Top panel: variability probability as a result of the statistical method for all known binaries in the MOCCA mock observation as a function of $\Delta v_{r} / 2$, a proxy for the projected radial velocity semiamplitude. Bottom panel: histograms of the variability probability for known single and binary stars in the MOCCA mock observation.

star. In the top panel of Fig. 7 the mean binary probability in relation to this semi-amplitude for all known binaries in the mock observation is presented, using a binning of 30 stars per data point. In view of the radial velocity uncertainties (see Fig. 4) the statistical method gives plausible results: for example $50 \%$ of all stars with a semi-amplitude around $10 \mathrm{~km} \mathrm{~s}^{-1}$ are recovered. The bottom panel of Fig. 7 shows histograms of the binary probability of the known single stars (in blue) and the known binary stars (in orange). We get a separation of the binary stars (peak at probability $\approx 1$ ) and single stars (peak at probability $\approx 0$ ). It shows the power of our approach, as it results in a binary distribution peaked at high probabilities and a single star distribution peaked at low probabilities. Using a threshold of $P=0.5(0.8)$ would result in a false-positive rate of 324/781 (44/617) stars. These rates agree with the expectations, since the method gives us a balance of false-positives and false-negatives at the $50 \%$ threshold, as mentioned before.

The statistical method yields a discovery fraction of $(22.1 \pm$ $1.8) \%$ for this mock observation. Compared with the true binary fraction in this mock observation of $25.9 \%$ we get a discovery efficiency of $85.2 \%$. This implies that we can expect to detect the vast majority of binary stars present in our observed MUSE sample.

\subsection{Application to the MUSE observations}

Using the statistical approach we obtained an observational binary frequency (discovery fraction) of $(17.1 \pm 1.9) \%$ within our MUSE FoV. The bottom panel of Fig. 6 shows the resulting binary probability of our stars in the sample. To determine the true binary frequency of the globular cluster NGC 3201 we have to overcome our observational biases. Our survey is a blind survey and we do not select our targets as in previous spectroscopic studies. Nevertheless, we still have a magnitude limit below which we cannot derive reliable radial velocities and we 
are confined to the MUSE FoV. In Fig. 5 the magnitude completeness of MUSE stars compared to the ACS globular cluster survey (Sarajedini et al. 2007) of NGC 3201 is shown (blue curve). After filtering (see Sect. 3), we end up with the magnitude completeness of the binary survey (orange curve). To get the binary frequency we have to divide the discovery fraction by the discovery efficiency. As described in Sect. 4, the discovery efficiency cannot be derived by simple assumptions (except for inclination of the binary system). Moreover, the efficiency strongly depends on the number of observations and the sampling per star. Figure 3 shows that our distribution of applicable observations is inhomogeneous and likewise is our time sampling (see Fig. 2).

We use the MOCCA mock observation to overcome these biases. Assuming the MOCCA simulation is realistic and comparable to our MUSE observation, we found a binary frequency of $(20.08 \pm 0.22) \%$ within our MUSE FoV, taking the discovery efficiency of Sect. 5.2 into account. We can now use the original MOCCA simulation and compare its true binary frequency of $8.72 \%$ with the true binary frequency of the mock observation $25.9 \%$ and get a factor of 0.336 . Applying this factor and the discovery efficiency it is possible to translate the MUSE discovery fraction into the total binary fraction of NGC 3201 to

\section{$(6.75 \pm 0.72) \%$.}

The main reason for this value being significantly lower than our observed binary frequency is the central increase in binaries due to mass segregation. Our value is in reasonable agreement with the binary fraction of the MOCCA simulation, $8.72 \%$, considering the assumptions that have to be made in Monte Carlo models. This highlights again that the MOCCA simulation that we selected represents an accurate model for NGC 3201.

We also verified to what extent our study is consistent with the results of Milone et al. (2012), who reported a core binary frequency of $(12.8 \pm 0.8) \%$. Without applying any selection function, the MOCCA simulation yields a binary fraction of $12.5 \%$ within the core radius of NGC 3201. Hence, our results appear to be consistent with the study of Milone et al. (2012) and the apparent differences (with respect to our discovery fraction of $(20.08 \pm 0.22) \%)$ can be attributed to selection effects.

\subsection{Primordial binaries}

We created different MOCCA simulations of NGC 3201 to get a comparable mock observation and thus binary fraction to our observations. The best matching MOCCA simulation (see Sect. 4) has an initial binary fraction of $50 \%$ which indicates that a large portion of primordial binaries is necessary to reproduce the current observations of NGC 3201.

Recent studies have shown that populations of cataclysmic variables and X-ray sources observed in several globular clusters can be better reproduced with globular cluster simulations that assume high primordial binary fractions (e.g. Rivera Sandoval et al. 2018; Cheng et al. 2018; Belloni et al. 2019). In addition, Leigh et al. (2015) and Cheng et al. (2019) found that a high primordial binary fraction is also necessary to reproduce the binary fraction outside the half-mass radius and the mass segregation we observe in Galactic globular clusters.

\subsection{Mass segregation}

Due to mass segregation it is expected that binary systems (which are in total more massive than single stars) will migrate towards the centre of a globular cluster (see Sect. 1). Thus the binary fraction should increase towards the cluster centre. In

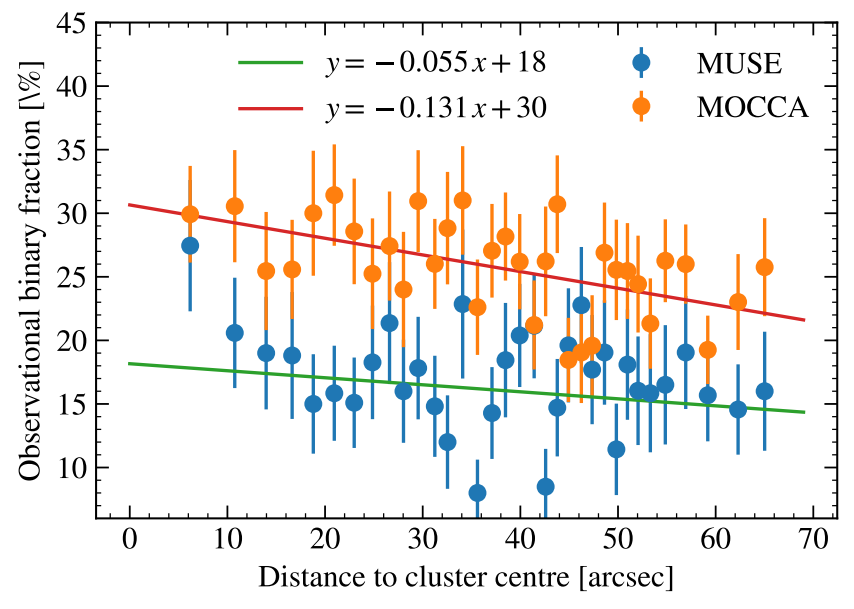

Fig. 8. Observational binary fraction of NGC 3201 in radial bins as a function of the cluster centre. Blue: MUSE bins with 100 stars per bin. Orange: MOCCA MUSE equivalent mock observation, same bins as MUSE.

Fig. 8 the observational binary discovery fraction in relation to the projected distance to the centre of the globular cluster is shown. Since we do not have true distances to the cluster centre, any observed radial trend is expected to be somewhat weaker than the underlying trend with true radii. Nevertheless, we found a mild increase in the binary fraction towards the cluster centre. The slope of a simple linear fit is $(-0.055 \pm 0.003) \%$ per arcsec with a Pearson correlation coefficient of -0.28 . Despite the observational biases and the limited radial distance to the cluster centre this result agrees with the theoretical expectations. Compared to the mock simulation, for which the same projected radial bins have been applied, the result is qualitatively the same. We like to stress that the original MOCCA simulation has a clear radial trend in the binary frequency (using three dimensional radial bins).

\section{Determination of orbital parameters}

Constraining the orbital properties (six standard Keplerian parameters) of a binary system with very few or imprecise radial velocity measurements is difficult. Naively one could assume that in dense globular clusters only hard binaries (with high binding energy and typically short period) could survive and should circulise their orbit during their lifetime. However, not only due to dynamical interactions, a significant number of binaries are expected to be on eccentric orbits (Hut et al. 1992). Methods like the generalised Lomb-Scargle periodogram (GLS; Zechmeister \& Kürster 2009) are good to find periods in unequally sampled data. GLS is good for finding periods in circular orbits, but tends to fail in eccentric configurations. The APOGEE team also faced the same challenges and created a tool named "THE JOKER" (Price-Whelan et al. 2017).

\subsection{THE JOKER}

THE JOKER is a custom Monte Carlo sampler for sparse or noisy radial velocity measurements of two-body systems and can produce posterior samples for orbital parameters even when the likelihood function is poorly behaved.

We follow the method of Price-Whelan et al. (2018) to find orbital solutions for our NGC 3201 stars. Our assumptions to use THE JOKER are accordingly: 

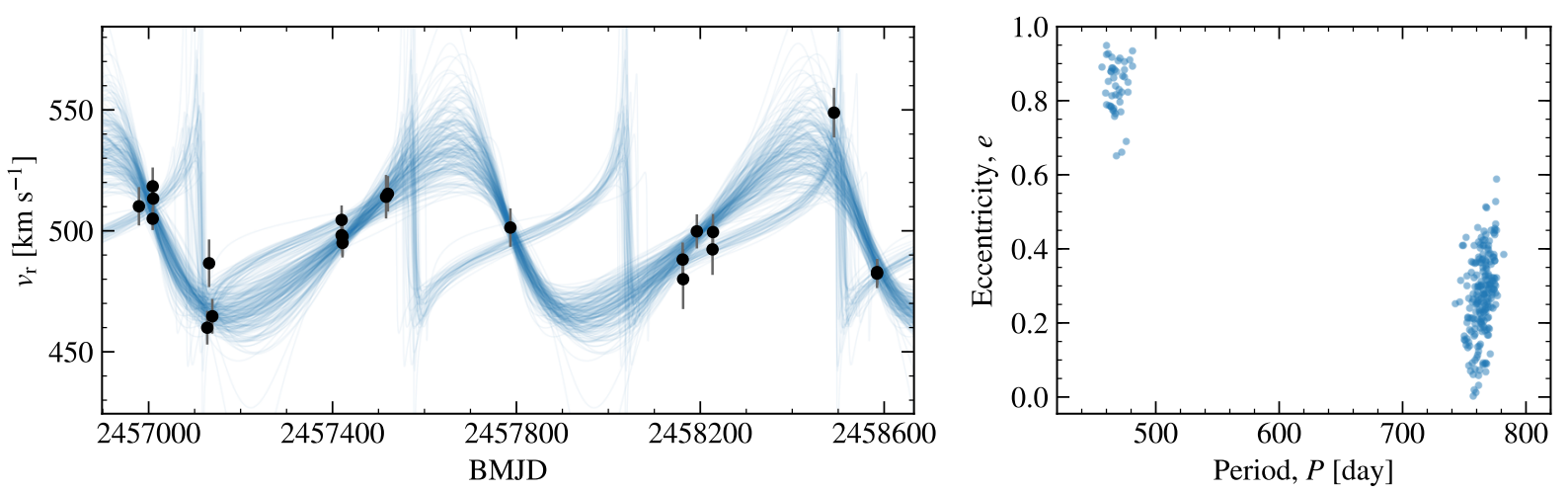

Fig. 9. Left: radial velocities (black data points) of the companion of the black hole candidate with ACS Id \#5132. The blue curves are the possible orbital solutions determined by THE JOKER for this star. Right: period-eccentricity plot of these samples.

- Two bodies: We assume the multiple star systems in NGC 3201 to consist of two stars or only two stars to be on the dominant timescale for the dynamics of the system (hierarchical multi-star system).

- SB1: The light of the binary components adds up in the MUSE spectrograph as a single source. We assume one of the two binary components does not contribute significantly to the spectrum (single-lined spectroscopic binary $=$ SB1). In the case both components have similar magnitudes (double-lined spectroscopic binary $=$ SB2) it is not possible for us to separate the components for typical radial velocity amplitudes, due to the low spectral resolution of MUSE. As mentioned in Sect. 4, the amplitude of SB2 systems depends on the flux ratio of the two binary components.

- Isolated Keplerian systems: The radial velocity variations are due to orbital motions and not caused by possible intrinsic variations (e.g. pulsations). Moreover, the cluster dynamics occurs on a longer timescale than the orbital motion in the binary system.

- Gaussian distributed uncertainties: We ensured all radial velocity uncertainties to be free from systematic effects, independent of each other and represent purely Gaussian noise (for details see Kamann et al. 2018, and Sect. 3.2).

We generated $2^{29}=536870912$ prior samples for the period range $0.3 \mathrm{~d}$ to $4096 \mathrm{~d}$. We requested 256 posterior samples for each star with a minimum of 5 observations and a minimum $50 \%$ probability to be a variable star. In our sample 515 met these conditions and could be processed with THE JOKER. If fewer than 200 posterior samples were found for one star, we used a dedicated Monte Carlo Markov chain (MCMC) run as described in Price-Whelan et al. (2017) to get 256 new samples. We analysed their posterior distributions and will focus on objects with unimodal or bimodal distributions in the following.

In Fig. 9 the result of this process for one example star (companion of a black hole candidate, more in Sect. 7.3) in our sample is presented. The 256 orbital solutions (in blue) represent the posterior likelihood distribution for this star and are in this case in principle two unique solutions with uncertainties. We define this solutions to be bimodal in orbital period. If in this example, only one solution (one cluster in the right panel of Fig. 9) would be present, we would define it as unimodal. In general, we have unimodal samples when the standard deviation of THE JOKER periods $P$ on logarithmic scale meets the criterion:

$\sigma_{\ln P}<0.5$.

To identify bimodal posterior samples we use a similar method as described in Price-Whelan et al. (2018): We use the $k$-means clustering with $k=2$ from the scikit-learn package (Pedregosa et al. 2011) to separate two clusters in the posterior samples in orbital period (like the two clusters in the right panel of Fig. 9). Each of the two clusters have to fulfil Eq. (7). We take the most probable (the one with more samples than the other) as the result for this bimodal posterior samples. Finally, we take the median and the standard deviation as uncertainty of all parameters from these results.

\subsection{Binary system properties}

The CMD in Fig. 10 is created using the newer HST UV globular cluster survey photometry of NGC 3201 (Nardiello et al. 2018; Piotto et al. 2015) which was matched to the photometry of the HST ACS globular cluster survey (Sarajedini et al. 2007; Anderson et al. 2008) we used as an input catalogue for the extraction of the spectra. The binary probability for each star we determined by our statistical method is colour-coded. As expected, many MS binaries positioned to the red of the MS are found by our method. Red giants brighter than the horizontal branch magnitude could not be found in binary systems in our sample. A good check for the statistical method are the chromospherically active binaries, which deviate in our CMD significantly from the MS (see annotation in CMD) and are very well confirmed by the method. More details about the other stellar types are presented in the following Sect. 7.

As described in Sect. 6.1 we analysed all stars with a 50\% variability probability. We found 95 stars from which 78 stars have unimodal and 17 stars bimodal posterior samples in orbital period. That means the period of these stars is well constrained but does not mean all Keplerian parameters are constrained similarly well. We present the results of THE JOKER in Table A.2 with the star position, magnitude, a selection of the fitted parameters (period, eccentricity, amplitude, visible mass), and the derived invisible minimum (companion) mass. The comment column contains additional information to individual stars as explained in the table notes. For a selection of interesting stars of this table we show the best Keplerian fit in Fig. A.1 and a blind random set in Fig. A.2. These plots show in the upper panel the radial velocities $v_{r}$ of an individual star from our final sample phase folded with the period from the best fitting model. The lower panel contains the residuals after subtracting this model from the data. In addition to the identifier (ACS Id), position and magnitude from the ACS catalogue (Sarajedini et al. 2007), we note the period $P$, eccentricity $e$, invisible minimum mass $M_{2}$, reduced $\chi^{2}$ of the best fitting model, and the comment in every plot for convenience. 


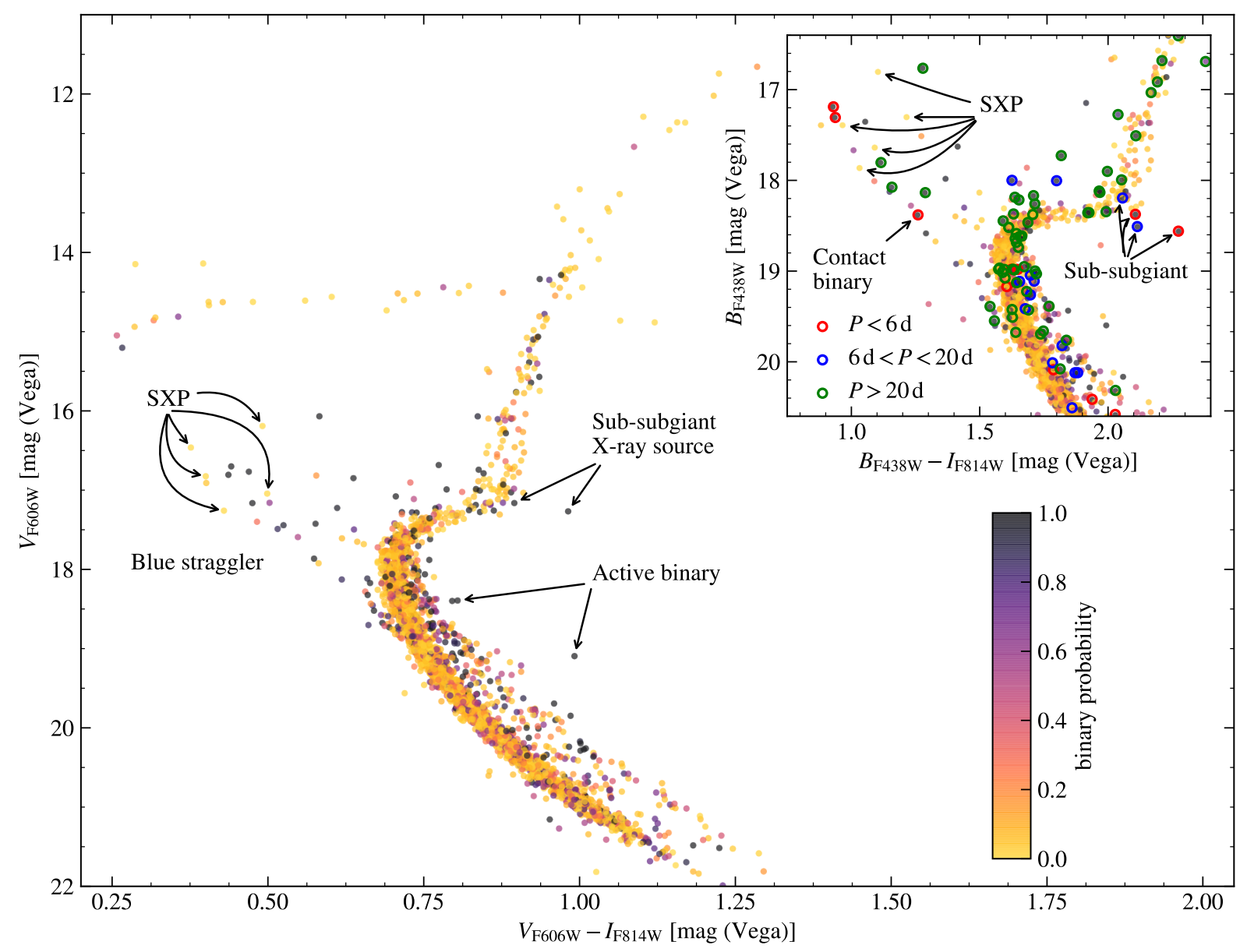

Fig. 10. Colour-magnitude diagram of the members of NGC 3201 created with the photometry taken from the HST UV globular cluster survey (Nardiello et al. 2018; Piotto et al. 2015). Colour-coded is the binary probability obtained by our statistical method. Large panel: full CMD of our sample using $V$ and $I$ equivalent filters. Small panel: detailed version of the MS turn-off CMD region using $B$ and $I$ equivalent filters. Additionally, the period $P$ range is indicated by coloured circles where THE JOKER was able to fit a well constrained Keplerian orbit.

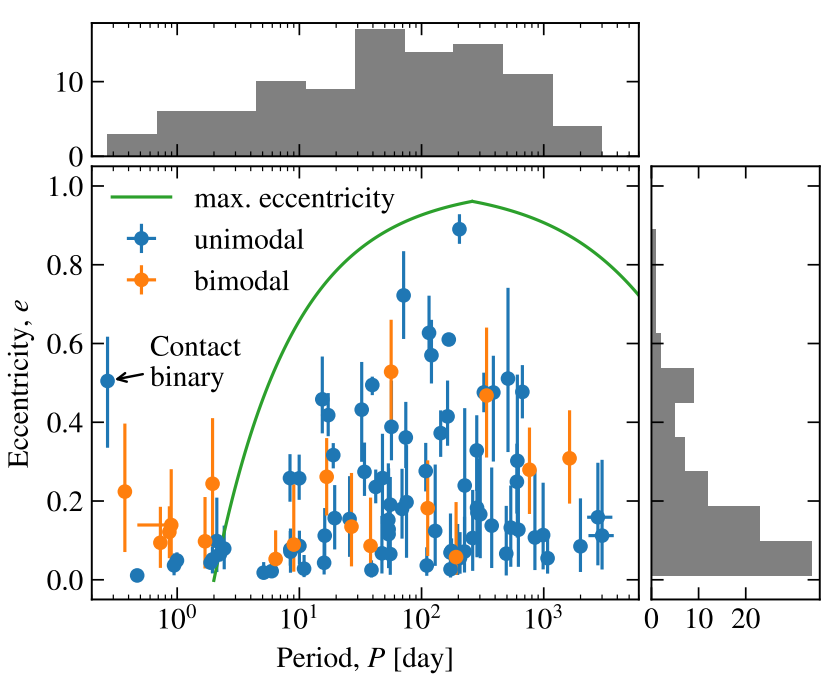

Fig. 11. Eccentricity-period plot of the well constrained binaries in NGC 3201. Binaries with unimodal (blue) and bimodal (orange) solutions in the posterior period sampling. The maximum eccentricity in green represents the theoretical limit (above a $2 \mathrm{~d}$ period) to which binary systems are stable (see Sect. 6.2).

For the first time Fig. 11 shows the eccentricity-period distribution of binaries in a globular cluster. We found binaries in a period range from $0.27 \mathrm{~d}$ to $3000 \mathrm{~d}$ with eccentricities from 0 to 0.9 . The eccentricity distribution is biased towards low eccentricities, because for these orbits fewer measurements are necessary to get a unique solution. In the figure we also show a maximum eccentricity $e_{\max }$ power law derived from a Maxwellian thermal eccentricity distribution

$e_{\max }(P)=1-\left(\frac{P}{2 \text { days }}\right)^{-2 / 3}$ for $P>2$ days

for a given period $P$ (Moe \& Di Stefano 2017). This represents the binary components having Roche lobe fill factors $\leq 70 \%$ at periastron. All binaries with $P<2 \mathrm{~d}$ should have circular orbits due to tidal forces. Additionally, we also take dynamical star interactions into account by using a limit on the orbital velocity at apoastron. The orbital velocity should always be higher than the central cluster dispersion of $5 \mathrm{~km} \mathrm{~s}^{-1}$ of NGC 3201 (Harris 1996, 2010 edition). This limit on eccentricity is dominant for periods $>260 \mathrm{~d}$. Except for the contact binary star (ACS Id: \#13108, see Table A.2) all stars behave accordingly. Note that when the eccentricity distribution of one star peaked at zero we still took the median like in all other distributions. Therefore the stars below $P<2$ are consistent within their uncertainty with $e=0$. Figure 11 also clearly shows that not all binaries in globular clusters have been circularised over the lifetime of a globular cluster. We, however, do not find high-eccentric long-period (e.g. $e>0.6$ and $P>300 \mathrm{~d}$ ) binaries within the eccentricity limit. 


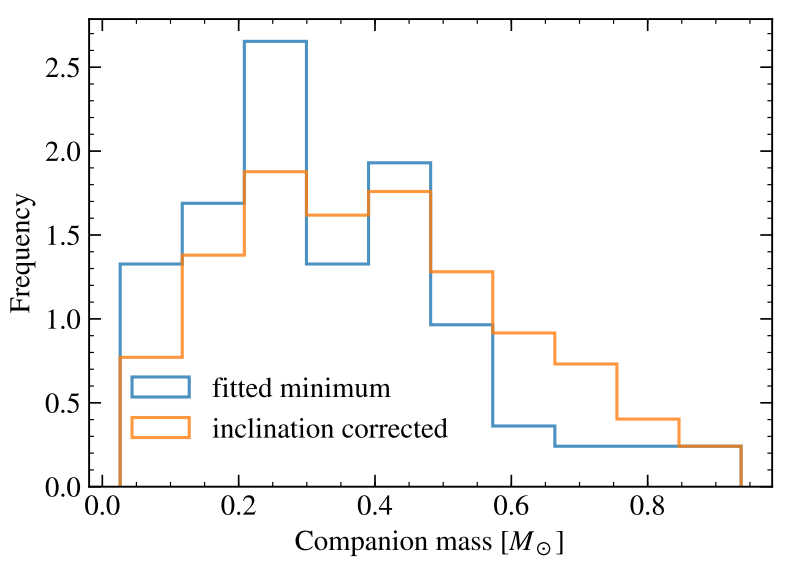

Fig. 12. Companion masses $\left(<1 M_{\odot}\right)$ of the well constrained binaries in NGC 3201 are shown. The blue histogram contains the fitted results (minimum masses) from THE JOKER, whereas the orange histogram contains the same results after applying a statistical correction for orbital inclination to obtain the actual companion mass distribution (see Sect. 6.2).

This could either be due to our observational bias or an effect which restricts the eccentricity even further.

Figure 12 shows the minimum companion masses of the well constrained binaries (blue histogram). In orange all masses are resampled 1000 times from their fitted mass divided by $\sin i$ to obtain the actual companion mass distribution. The inclination $i$ is taken from a sinusoidal detection probability distribution between 0 to $\pi / 2$ with a cutoff mass of $0.8 M_{\odot}$ (roughly maximum normal stellar mass in NGC 3201). This results in a linear anti-correlation between the companion mass and the frequency of binaries having this companion mass. As explained in Sect. 4 we have a bias towards binaries with both components having different luminosities. Milone et al. (2012) found a flat mass ratio distribution throughout all clusters above a mass ratio $>0.5$. The pairing fraction of our binaries is beyond the scope of this paper, since we would need to correct for the luminosity ratio effect and the selection function of THE JOKER, which is currently unknown in our case.

Noteworthy is that all red giant binaries in our sample with well constrained orbits have periods larger than $100 \mathrm{~d}$. This corresponds to a minimum semi-major axis of $\gtrsim 0.5 \mathrm{AU}$ and is consistent with not having Roche-lobe overflow in the binary system.

\section{Peculiar objects in NGC 3201}

\subsection{Blue straggler stars and SX Phoenicis-type stars}

Our sample of NGC 3201 contains 40 blue stragglers with sufficient observations and signal to noise. From these stars, 23 are most likely in binary systems while 17 are not (including the SX Phoenicis-type stars assumed to be single stars). This observational binary fraction of $(57.5 \pm 7.9) \%$ is much higher than the one of all cluster stars or even the Milky Way field stars (see Sect. 1). Our ratio of binaries to single blue straggler stars $R_{\mathrm{B} / \mathrm{S}}=1.35$ in the core of NGC 3201 is significantly higher than the prediction by Hypki \& Giersz (2017) of $R_{\mathrm{B} / \mathrm{S}} \sim 0.4$, but could also be explained by an overabundance of the more massive blue straggler binaries (compared to single blue stragglers) due to mass segregation. Nevertheless, this high binary fraction strongly suggests that most of the blue stragglers in our sample were formed within a binary system. As in Sect. 2 explained, a cluster member needs additional mass to become a blue straggler. Two formation scenarios are consistent with this high binary fraction: (1) mass transfer within a triple star system (e.g. Antonini et al. 2016), (2) stellar mergers induced by stellar interactions of binary systems with other binaries or single stars (e.g. Leonard 1989; Fregeau et al. 2004).

From these 23 blue straggler binaries THE JOKER could find 11 highly constrained solutions, three are extremely hard binaries $(<1 \mathrm{~d})$ with a minimum companion mass ranging from $0.11 M_{\odot}$ to $0.24 M_{\odot}$ and six relatively wide $(>100 \mathrm{~d})$ with minimum companion masses ranging from $0.35 M_{\odot}$ to $0.67 M_{\odot}$. The remaining 2 blue stragglers are in between the period and companion mass range (see stars in Table A.2 with comment "BSS" for more details). Figure 10 shows the blue stragglers and their period range in a CMD. We also identified five blue straggler stars as known SX Phoenicis-type variable stars in the Catalogue of Variable Stars in Galactic Globular Clusters (Clement et al. 2001; Clement 2017) and one of them as a SX Phoenicis candidate in Arellano Ferro et al. (2014). We also see their pulsations (similar to the RR Lyrae-type stars) as a radial velocity signal up to amplitudes of $18 \mathrm{~km} \mathrm{~s}^{-1}$. We cannot distinguish between the pulsation signal and a possible signal induced by a binary companion, thus we consider these stars to be single stars and the velocity variability solely explained by radial pulsations.

Mass transfer in a relatively wide blue straggler binary system is almost impossible. Thus, only the three hard binaries probably result from this process. It is obvious that mass transfer is taking place in the contact binary system with ACS Id $\# 13108$. On the other hand, head-on collisions of single stars would result in single blue stragglers and are extremely unlikely in a low-density cluster such as NGC 3201. Therefore, it is more likely that a high percentage of the blue stragglers in our sample result from coalescence in systems with $\geq 3$ stars. The complex behaviour of such systems favours configurations that result in the coalescence of two of its members (e.g. Leonard 1989; Fregeau et al. 2004; Antonini et al. 2016). If more than two stars in the formation of blue stragglers are involved, this could also mean that hard blue straggler binary systems could have a third outer component inducing an additional, much weaker signal. We could not identify such a signal in our data. For example, the star with ACS Id \#12746 (see radial velocity signal in Fig. A.1) is the most central hard blue straggler binary in our sample with 63 observations, but its radial velocity curve does not contain any significant additional signal.

The projected spin velocities $(V \sin i)$ of blue stragglers have been suggested as a possibility to infer their ages and origins (e.g. Leiner et al. 2018). $V \sin i$ measurements of blue stragglers in NGC 3201 have been performed by Simunovic \& Puzia (2014) and cross-matching their sample with our data results in a subset of five blue stragglers with spin velocities ranging from $(34.6 \pm 1.6) \mathrm{km} \mathrm{s}^{-1}$ to $(135.2 \pm 3.5) \mathrm{km} \mathrm{s}^{-1}$. We note that all of them are binary systems. However, no clear picture emerges regarding a possible relation between spin velocity and orbital properties.

As introduced, SX Phoenicis-type stars are most likely formed in binary evolution. That is why it is possible for them to have a companion, but the companions radial velocity signal would be hidden in the pulsation signal.

\subsection{Sub-subgiant stars}

We found four SSG stars ${ }^{4}$ within our MUSE FoV (see position in Fig. 10). Assuming the radial velocity changes are only

\footnotetext{
4 The four SSGs in our sample could also be defined as red stragglers,
} since they are at the boundary of both definitions in Geller et al. (2017a). 
Keplerian (and not by pulsations), THE JOKER could determine unique Keplerian solutions for all of them (see stars in Table A.2 with comment "SSG").

Fortunately the SSG with ACS Id \#14749 (see also plot in Fig. A.1) is a known detached eclipsing binary with a period of $10.0037 \mathrm{~d}$ (Kaluzny et al. 2016) and we found the same period $(10.006 \pm 0.002) \mathrm{d}$ with our method. But this also means the system is observed edge-on and our companion mass is its true mass. The visible star shows a radial velocity amplitude of $(42.9 \pm 1.5) \mathrm{km} \mathrm{s}^{-1}$ with a very low eccentricity of $(0.09 \pm 0.07)$, and a companion mass of $(0.53 \pm 0.04) M_{\odot}$ for an assumed primary mass of $(0.82 \pm 0.05) M_{\odot}$. That makes a system mass of $1.35 M_{\odot}$ and considering mass transfer, one possible pathway could be towards the blue straggler region (Leiner et al. 2017). Compared to our best fitting model spectrum (and to a well fitted $\mathrm{H} \beta$ line) all spectra of this star show a partially filled-in $\mathrm{H} \alpha$ absorption line (the absorption is not as deep as expected, see Fig. A.3 as an example). This could mean that mass transfer is already underway. In this case this could be used to infer an accretion rate, but is beyond the scope of this paper.

The two short period ( $<6 \mathrm{~d}, \# 22692, \# 13438)$ SSGs show X-ray emission according to the November 2017 pre-release of the Chandra Source Catalog Release 2.0 (Evans et al. 2010). The SSG (\#22692) with the $5.1 \mathrm{~d}$ period actually has significant varying $\mathrm{H} \alpha$ emission lines in most of our spectra. One spectrum of this star with $\mathrm{H} \alpha$ in emission is presented in Fig. A.4. The maximum emission line is twice as strong as the minimum line compared to the continuum. Again, it would be interesting to calculate the accretion rate for this case, but is beyond the scope of this paper. The SSG (\#13438) with the $5.9 \mathrm{~d}$ period has partially filled-in $\mathrm{H} \alpha$ absorption lines in all spectra like the eclipsing SSG described before. One spectrum of this star with the filled-in $\mathrm{H} \alpha$ line is shown in Fig. A.3. Both short period SSGs have a similar semi-amplitude of $\sim 37 \mathrm{~km} \mathrm{~s}^{-1}$, no eccentricity and a minimum companion mass of $(0.35 \pm 0.03) M_{\odot}$.

The SSG (\#11405) has with $17.2 \mathrm{~d}$ the longest period, unlike the other stars a relatively high eccentricity of 0.4 and a low minimum companion mass of $0.15 M_{\odot}$.

The detection of a dozen more SSGs and RSs in our MUSE globular cluster sample will be published in the emission line catalogue of Göttgens et al. (2019b).

\subsection{Black hole candidates}

Here we report our new measurements for the stellar-mass black hole $(\mathrm{BH})$ candidate in NGC 3201 previously published in Giesers et al. (2018). The additional observations perfectly match the known orbital model and confirm the $\mathrm{BH}$ minimum mass to be $(4.53 \pm 0.21) M_{\odot}$. The deviation to the previously minimum mass of $(4.36 \pm 0.41) M_{\odot}$ is within the uncertainties. Furthermore, the observations fill in the apoapsis which was missed by previous observations (see \#12560 in Fig. A.1) and exclude other possible orbital solutions. We would like to emphasise that this star has been analysed with THE JOKER and MCMC like all other stars, without the effort invested in it as in Giesers et al. (2018).

In our full sample of NGC 3201 we detected two additional BH candidates with solutions from the fit of THE JOKER. Table A.2 shows the resulting properties of these binary systems with ACS Id \#21859 and \#5139. Both systems are faint with extracted spectra of $S / N \leq 10$. Remarkable is the system (\#21859) with a unique solution and a semi-amplitude of about $300 \mathrm{~km} \mathrm{~s}^{-1}$, we cannot explain the radial velocity curve shown in Fig. A.1 with other explanations than an unseen companion with a minimum mass of $(7.68 \pm 0.50) M_{\odot}$. The other system (\#5132) has two solutions with the more probable ( $82 \%$ probability) minimum companion mass of $(4.40 \pm 2.82) M_{\odot}$ and the less probable of $1.10 \pm 0.20 M_{\odot}$. This candidate is not well constrained and needs more observations to be confirmed as a BH. To our knowledge, both sources do not have an X-ray or radio counterpart. This is remarkable in the case of \#21859, as the presence of a partially filled-in $\mathrm{H} \alpha$ line in the MUSE spectra suggests that accretion is present. We show a combination of all spectra shifted to rest-frame in Fig. A.5.

In light of the recent results based on Monte Carlo simulations, it appears feasible that NGC 3201 hosts several BHs in binaries with MS stars. Both Kremer et al. (2018) and Askar et al. (2018a) predict the presence of at least $\sim 100 \mathrm{BHs}$ in the cluster, with tens of them in binaries with other BHs or bright companions. More specifically, in the best model of Kremer et al. (2018), four $\mathrm{BHs}$ have MS-star companions. Interestingly, all of those systems show eccentricities $\gtrsim 0.6$, add odds with the new candidates that we discovered (cf. Table A.2). On the other hand, the semi-major axes of our candidates of $0.067 \mathrm{AU}$ and $2.804 \mathrm{AU}$ are within the range of semi-major-axis values that Kremer et al. (2018) found in their best model of NGC 3201 (see their Fig. 3).

Our MOCCA snapshot at $12 \mathrm{Gyr}$ contains 43 BHs with 33 single $\mathrm{BHs}, 2 \mathrm{BH}-\mathrm{BH}$ binaries, and $6 \mathrm{BH}$-star binaries. The median mass of all $\mathrm{BHs}$ is $16.8 M_{\odot}$ within a range of $3.7 M_{\odot}$ to $78 M_{\odot}$. Two $\mathrm{BH}$-star binaries and 5 single $\mathrm{BHs}$ are in the probable mass region of the $\mathrm{BH}$ candidates we discussed here. The total number of BHs is lower than the previous predictions, but the number of BHs with detectable companions is consistent with our observations throughout all models.

\section{Conclusions and outlook}

We elaborated the first binary study from our MUSE survey of 27 Galactic globular clusters in the core of NGC 3201 and showed the variety of results our blind multi-epoch spectroscopic observations reveal. Since modelling a globular cluster contains many imponderables, we developed a statistical method - applicable to any time-variant measurements with inhomogeneous sampling and uncertainties (not only radial velocities) - which can distinguish between a constant and varying signal (like in single and binary stars). We determined an observational binary frequency of $(20.08 \pm 0.22) \%$ using this statistical method. Based on a comparison with an advanced MOCCA simulation of NGC 3201, we calculated a total binary fraction of $(6.75 \pm 0.72) \%$ for all stars in the whole cluster. We confirmed the trend of an increasing binary fraction towards the cluster centre due to mass segregation. We found a significantly higher binary fraction $(57.5 \pm 7.9) \%$ of blue straggler stars compared to the cluster, indicating that the formation of blue stragglers in the core of NGC 3201 is related to binary evolution. For the first time, we presented well constrained Keplerian orbit solutions for a significant amount of stars (95) using the Monte Carlo tool THE JOKER. Eleven of these are blue straggler stars very likely in binary systems and shed light on the properties of these systems. We conclude that both the mass transfer formation scenario and the collisional formation scenario of blue stragglers are present in our data. Collision means in this case the coalescence of two stars during a binary-binary or binary-single encounter. We found four SSG stars by connecting our MUSE spectroscopy with HST photometry and X-ray observations. Fortunately, we got definite Keplerian solutions for all of them and have insights into their properties for the first time in a globular cluster. Finally, we presented three stellar-mass BH candidates, from which one is already published (Giesers et al. 2018) and one with a minimum mass of 
$(7.68 \pm 0.50) M_{\odot}$ is clearly above a single and even binary neutron star companion mass limit. In total, these BH candidates in binary systems with MS stars would strongly support the hypothesis that NGC 3201 has an extensive BH population of up to hundred more BHs (Kremer et al. 2018; Askar et al. 2018a). This cluster, and maybe other globular clusters as well, could be a significant source of gravitational waves.

In a following paper we will present binary fractions in the context of multiple stellar populations within NGC 3201. We will continue to observe $\omega$ Cen and 47 Tuc to have a comparable amount of epochs and will end up with a magnitude more stars per cluster compared to NGC 3201. Finally, we will publish the binary fractions of all clusters in our survey and try to find correlations with cluster parameters.

Acknowledgements. We thank Arash Bahramian, Nate Bastian, Robert Mathieu, Wolfram Kollatschny, Stan Lai, and Mark Gieles for helpful discussions. BG, SD, SK and PMW acknowledge support from the German Ministry for Education and Science (BMBF Verbundforschung) through grants 05A14MGA, 05A17MGA, 05A14BAC, and 05A17BAA. AA is supported by the Carl Tryggers Foundation for Scientific Research through the grant CTS 17:113. SK gratefully acknowledges funding from a European Research Council consolidator grant (ERC-CoG-646928- Multi-Pop). JB acknowledges support by FCT/MCTES through national funds by grant UID/FIS/04434/2019 and through Investigador FCT Contract No. IF/01654/2014/CP1215/CT0003. This research is supported by the German Research Foundation (DFG) with grants DR 281/351 and KA 4537/2-1. Based on observations made with ESO Telescopes at the La Silla Paranal Observatory under programme IDs 094.D-0142, 095.D-0629, 096.D-0175，097.D-0295，098.D-0148，0100.D-0161，0101.D-0268，0102.D0270, and 0103.D-0204. Based on observations made with the NASA/ESA Hubble Space Telescope, obtained from the data archive at the Space Telescope Science Institute. STScI is operated by the Association of Universities for Research in Astronomy, Inc. under NASA contract NAS 5-26555. Supporting data for this article is available at: http://musegc.uni-goettingen.de.

\section{References}

Anderson, J., Sarajedini, A., Bedin, L. R., et al. 2008, AJ, 135, 2055 Antonini, F., Chatterjee, S., Rodriguez, C. L., et al. 2016, ApJ, 816, 65 Arca Sedda, M., Askar, A., \& Giersz, M. 2018, MNRAS, 479, 4652 Arellano Ferro, A., Ahumada, J. A., Calderón, J. H., \& Kains, N. 2014, Rev. Mex. Astron. Astrofis., 50, 307

Askar, A., Szkudlarek, M., Gondek-Rosińska, D., Giersz, M., \& Bulik, T. 2017, MNRAS, 464, L36

Askar, A., Arca Sedda, M., \& Giersz, M. 2018a, MNRAS, 478, 1844

Askar, A., Giersz, M., Pych, W., \& Dalessandro, E. 2018b, MNRAS, 475, 4170

Askar, A., Askar, A., Pasquato, M., \& Giersz, M. 2019, MNRAS, 485, 5345

Baldwin, A. T., Watkins, L. L., van der Marel, R. P., et al. 2016, ApJ, 827, 12

Baumgardt, H. 2017, MNRAS, 464, 2174

Baumgardt, H., \& Hilker, M. 2018, MNRAS, 478, 1520

Belczynski, K., Kalogera, V., \& Bulik, T. 2002, ApJ, 572, 407

Belloni, D., Askar, A., Giersz, M., Kroupa, P., \& Rocha-Pinto, H. J. 2017, MNRAS, 471, 2812

Belloni, D., Giersz, M., Rivera Sandoval, L. E., Askar, A., \& Ciecielåg, P. 2019, MNRAS, 483, 315

Bressan, A., Marigo, P., Girardi, L., et al. 2012, MNRAS, 427, 127 Chatterjee, S., Rodriguez, C. L., \& Rasio, F. A. 2017, ApJ, 834, 68 Cheng, Z., Li, Z., Xu, X., \& Li, X. 2018, ApJ, 858, 33

Cheng, Z., Li, Z., Li, X., Xu, X., \& Fang, T. 2019, ApJ, 876, 59

Clement, C. M. 2017, VizieR Online Data Catalog: V/150

Clement, C. M., Muzzin, A., Dufton, Q., et al. 2001, AJ, 122, 2587

Cohen, R. E., \& Sarajedini, A. 2012, MNRAS, 419, 342

Dotter, A., Sarajedini, A., Anderson, J., et al. 2010, ApJ, 708, 698

Duchêne, G., \& Kraus, A. 2013, ARA\&A, 51, 269

Duquennoy, A., \& Mayor, M. 1991, A\&A, 248, 485

Evans, I. N., Primini, F. A., Glotfelty, K. J., et al. 2010, ApJS, 189, 37

Ferraro, F. R., Paltrinieri, B., Fusi Pecci, F., et al. 1997, A\&A, 324, 915

Fiorentino, G., Lanzoni, B., Dalessandro, E., et al. 2014, ApJ, 783, 34

Fregeau, J. M., Cheung, P., Portegies Zwart, S. F., \& Rasio, F. A. 2004, MNRAS, 352, 1

Fregeau, J. M., Ivanova, N., \& Rasio, F. A. 2009, ApJ, 707, 1533
Geller, A. M., \& Mathieu, R. D. 2011, Nature, 478, 356

Geller, A. M., Leiner, E. M., Bellini, A., et al. 2017a, ApJ, 840, 66 Geller, A. M., Leiner, E. M., Chatterjee, S., et al. 2017b, ApJ, 842, 1 Giersz, M. 1998, MNRAS, 298, 1239

Giersz, M., Heggie, D. C., Hurley, J. R., \& Hypki, A. 2013, MNRAS, 431, 2184

Giesers, B., Dreizler, S., Husser, T.-O., et al. 2018, MNRAS, 475, L15 Goodman, J., \& Hut, P. 1989, Nature, 339, 40

Göttgens, F., Weilbacher, P. M., Roth, M. M., et al. 2019a, A\&A, 626, A69 Göttgens, F., Husser, T. O., Kamann, S., et al. 2019b, A\&A, 631, A118 Harris, W. E. 1996, AJ, 112, 1487

Hénon, M. H. 1971, Ap\&SS, 14, 151

Hobbs, G., Lorimer, D. R., Lyne, A. G., \& Kramer, M. 2005, MNRAS, 360, 974

Hong, J., Vesperini, E., Askar, A., et al. 2018, MNRAS, 480, 5645

Hurley, J. R., Pols, O. R., \& Tout, C. A. 2000, MNRAS, 315, 543

Hurley, J. R., Tout, C. A., \& Pols, O. R. 2002, MNRAS, 329, 897

Hurley, J. R., Aarseth, S. J., \& Shara, M. M. 2007, ApJ, 665, 707

Husser, T.-O., Wende-von Berg, S., Dreizler, S., et al. 2013, A\&A, 553, A6

Husser, T.-O., Kamann, S., Dreizler, S., et al. 2016, A\&A, 588, A148

Husser, T. O., Latour, M., Brinchmann, J., et al. 2019, A\&A, submitted

Hut, P., McMillan, S., Goodman, J., et al. 1992, PASP, 104, 981

Hypki, A., \& Giersz, M. 2013, MNRAS, 429, 1221

Hypki, A., \& Giersz, M. 2017, MNRAS, 466, 320

Ivanova, N., Belczynski, K., Fregeau, J. M., \& Rasio, F. A. 2005, MNRAS, 358, 572

Joshi, K. J., Rasio, F. A., \& Portegies Zwart, S. 2000, ApJ, 540, 969

Kaluzny, J., Rozyczka, M., Thompson, I. B., et al. 2016, Acta Astron., 66, 31

Kamann, S., Wisotzki, L., \& Roth, M. M. 2013, A\&A, 549, A71

Kamann, S., Husser, T.-O., Dreizler, S., et al. 2018, MNRAS, 473, 5591

Kim, C., McNamara, D. H., \& Christensen, C. G. 1993, AJ, 106, 2493

King, I. R. 1966, AJ, 71, 64

Kremer, K., Ye, C. S., Chatterjee, S., Rodriguez, C. L., \& Rasio, F. A. 2018, ApJ, 855, L15

Kroupa, P. 2001, MNRAS, 322, 231

Latour, M., Husser, T. O., Giesers, B., et al. 2019, A\&A, 631, A14

Leigh, N. W. C., Giersz, M., Marks, M., et al. 2015, MNRAS, 446, 226

Leiner, E., Mathieu, R. D., \& Geller, A. M. 2017, ApJ, 840, 67

Leiner, E., Mathieu, R. D., Gosnell, N. M., \& Sills, A. 2018, ApJ, 869, L29

Leonard, P. J. T. 1989, AJ, 98, 217

McLaughlin, D. E., \& van der Marel, R. P. 2005, ApJS, 161, 304

Milone, A. P., Piotto, G., Bedin, L. R., et al. 2012, A\&A, 540, A16

Moe, M., \& Di Stefano, R. 2017, ApJS, 230, 15

Morscher, M., Pattabiraman, B., Rodriguez, C., Rasio, F. A., \& Umbreit, S. 2015, ApJ, 800, 9

Nardiello, D., Libralato, M., Piotto, G., et al. 2018, MNRAS, 481, 3382

Pattabiraman, B., Umbreit, S., Liao, W.-K., et al. 2013, ApJS, 204, 15

Pedregosa, F., Varoquaux, G., Gramfort, A., et al. 2011, J. Mach. Learn. Res., 12,2825

Piotto, G., Milone, A. P., Bedin, L. R., et al. 2015, AJ, 149, 91

Price-Whelan, A. M., Hogg, D. W., Foreman-Mackey, D., \& Rix, H.-W. 2017, ApJ, 837, 20

Price-Whelan, A. M., Hogg, D. W., Rix, H.-W., et al. 2018, AJ, 156, 18

Rivera Sandoval, L. E., van den Berg, M., Heinke, C. O., et al. 2018, MNRAS, 475,4841

Rodriguez, C. L., Chatterjee, S., \& Rasio, F. A. 2016, Phys. Rev. D, 93, 084029

Sarajedini, A., Bedin, L. R., Chaboyer, B., et al. 2007, AJ, 133, 1658

Simunovic, M., \& Puzia, T. H. 2014, ApJ, 782, 49

Sollima, A., Beccari, G., Ferraro, F. R., Fusi Pecci, F., \& Sarajedini, A. 2007, MNRAS, 380, 781

Sollima, A., Lanzoni, B., Beccari, G., Ferraro, F. R., \& Fusi Pecci, F. 2008, A\&A, 481, 701

Sommariva, V., Piotto, G., Rejkuba, M., et al. 2009, A\&A, 493, 947

Strader, J., Chomiuk, L., Maccarone, T. J., Miller-Jones, J. C. A., \& Seth, A. C. 2012, Nature, 490, 71

Tonry, J., \& Davis, M. 1979, AJ, 84, 1511

Tremou, E., Strader, J., Chomiuk, L., et al. 2018, ApJ, 862, 16

Wang, L., Spurzem, R., Aarseth, S., et al. 2016, MNRAS, 458, 1450

Weilbacher, P. M., Streicher, O., Urrutia, T., et al. 2012, in Software and Cyberinfrastructure for Astronomy II, Proc. SPIE, 8451, 84510B

Weilbacher, P. M., Streicher, O., Urrutia, T., et al. 2014, in Astronomical Data Analysis Software and Systems XXIII, eds. N. Manset, P. Forshay, et al., ASP Conf. Ser., 485, 451

Wendt, M., Husser, T.-O., Kamann, S., et al. 2017, A\&A, 607, A133

Zechmeister, M., \& Kürster, M. 2009, A\&A, 496, 577

Zocchi, A., Gieles, M., \& Hénault-Brunet, V. 2019, MNRAS, 482, 4713 

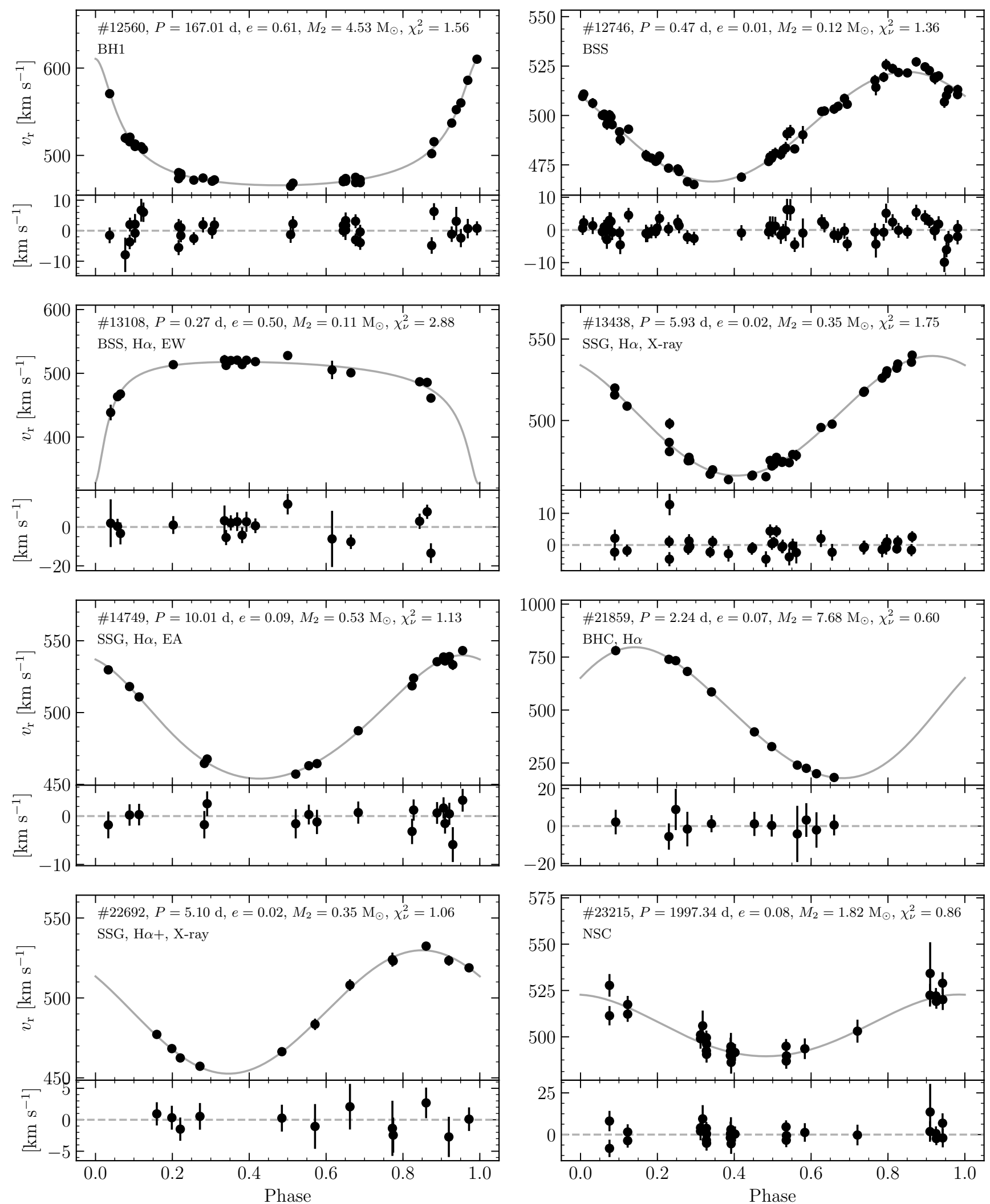

Fig. A.1. Selection of interesting results from THE JOKER sorted by ACS Id. The upper panel of every plot shows the radial velocities of the star from our final sample phase folded with the period from the best-fitting model. (Sometimes the uncertainties appear smaller as the data points.) The best-fitting model is plotted with the continues line. The lower panel contains the residuals after subtracting this model from the data. We note the source number, period $P$, eccentricity $e$, invisible mass $M_{2}$ and reduced $\chi^{2}$ of the best fitting model in every plot. See Table 1 for more information to individual stars. 

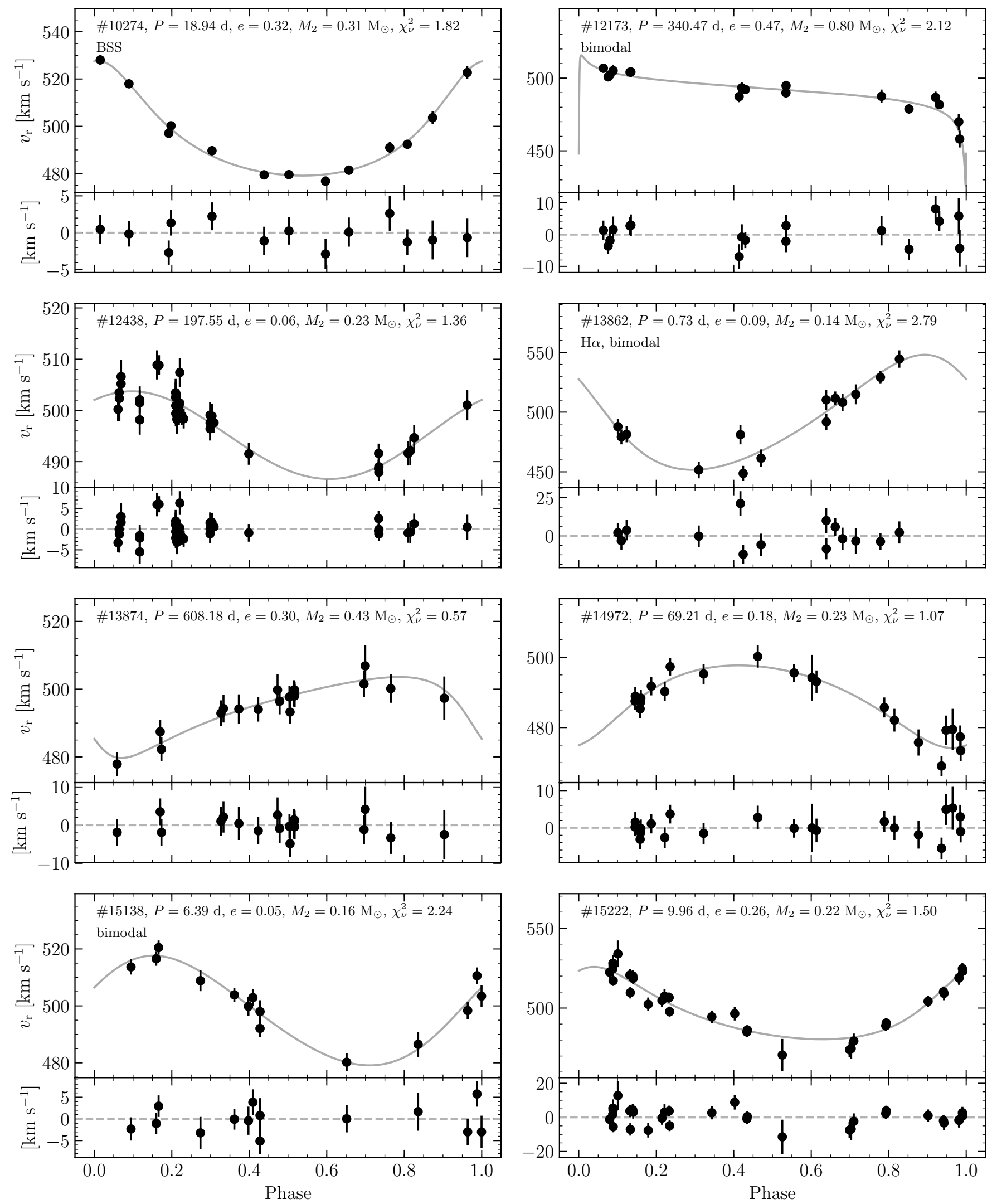

Fig. A.2. Same as Fig. A.1 for a random selection of results from THE JOKER. 


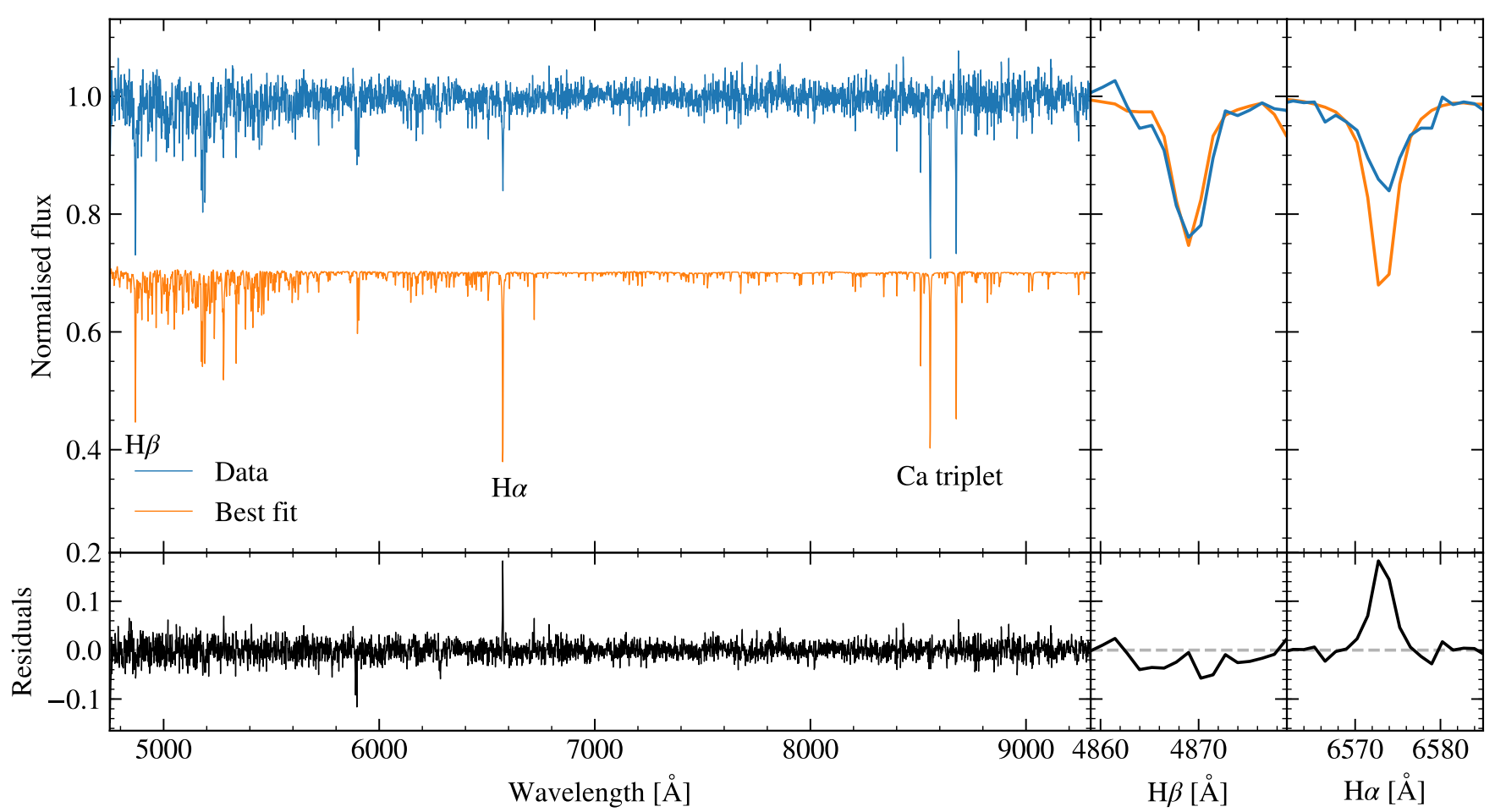

Fig. A.3. Spectrum of the sub-subgiant with ACS Id \#13438. Top panel: one normalised observed spectrum in blue and the best-fitting PHOENIX spectrum offset by 0.3 in orange. Bottom panel: residuals after subtracting the best fit from the data. Right panels: zoom at the $\mathrm{H} \beta$ and $\mathrm{H} \alpha$ line with a filled-in observed $\mathrm{H} \alpha$ line in contrast to the best fit (not offset).

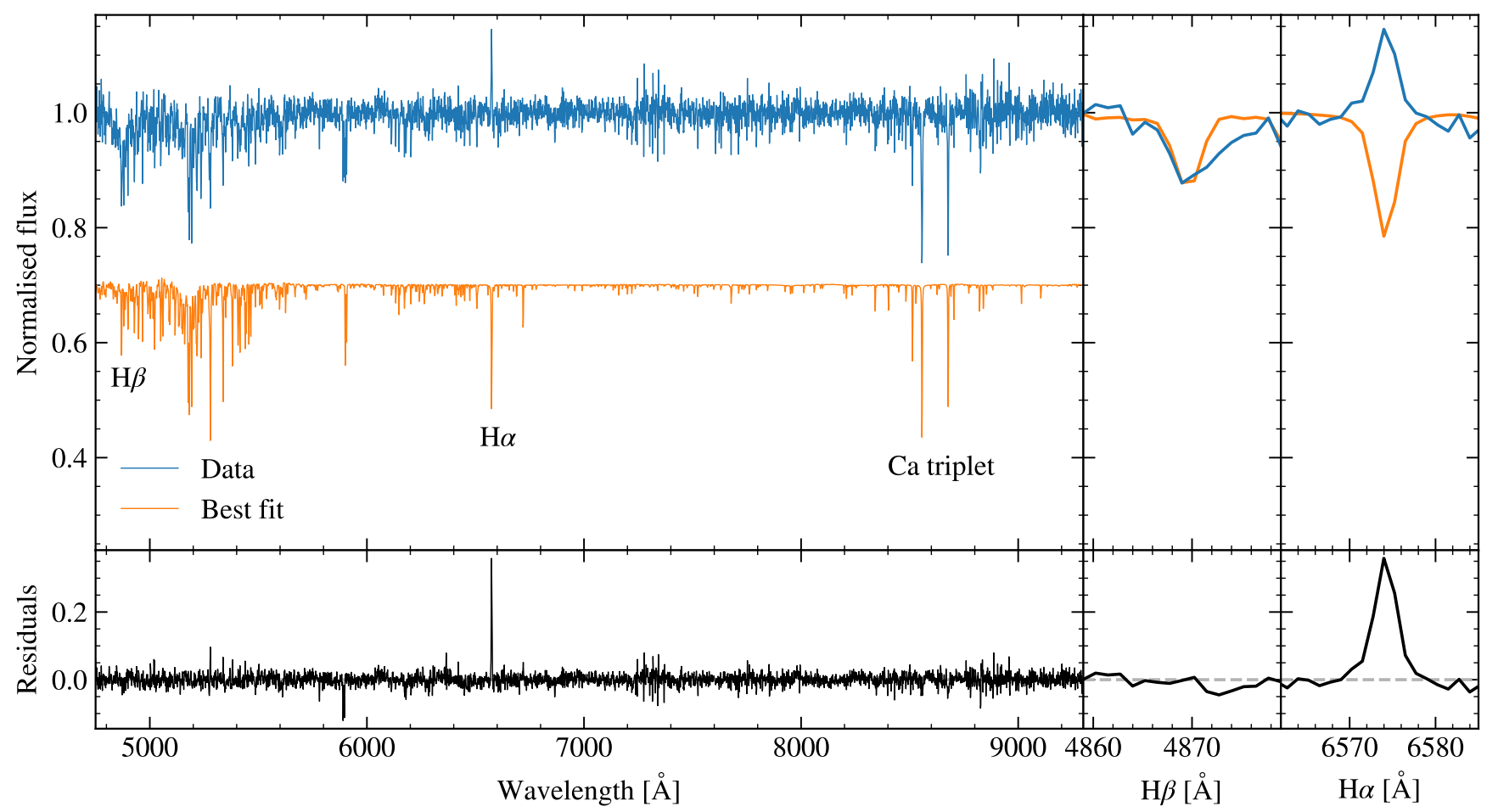

Fig. A.4. Same as Fig. A.3 for the sub-subgiant with ACS Id \#22692. Note that in contrast to star \#13438 (cf. Fig. A.3), this star shows an observed $\mathrm{H} \alpha$ emission line. 


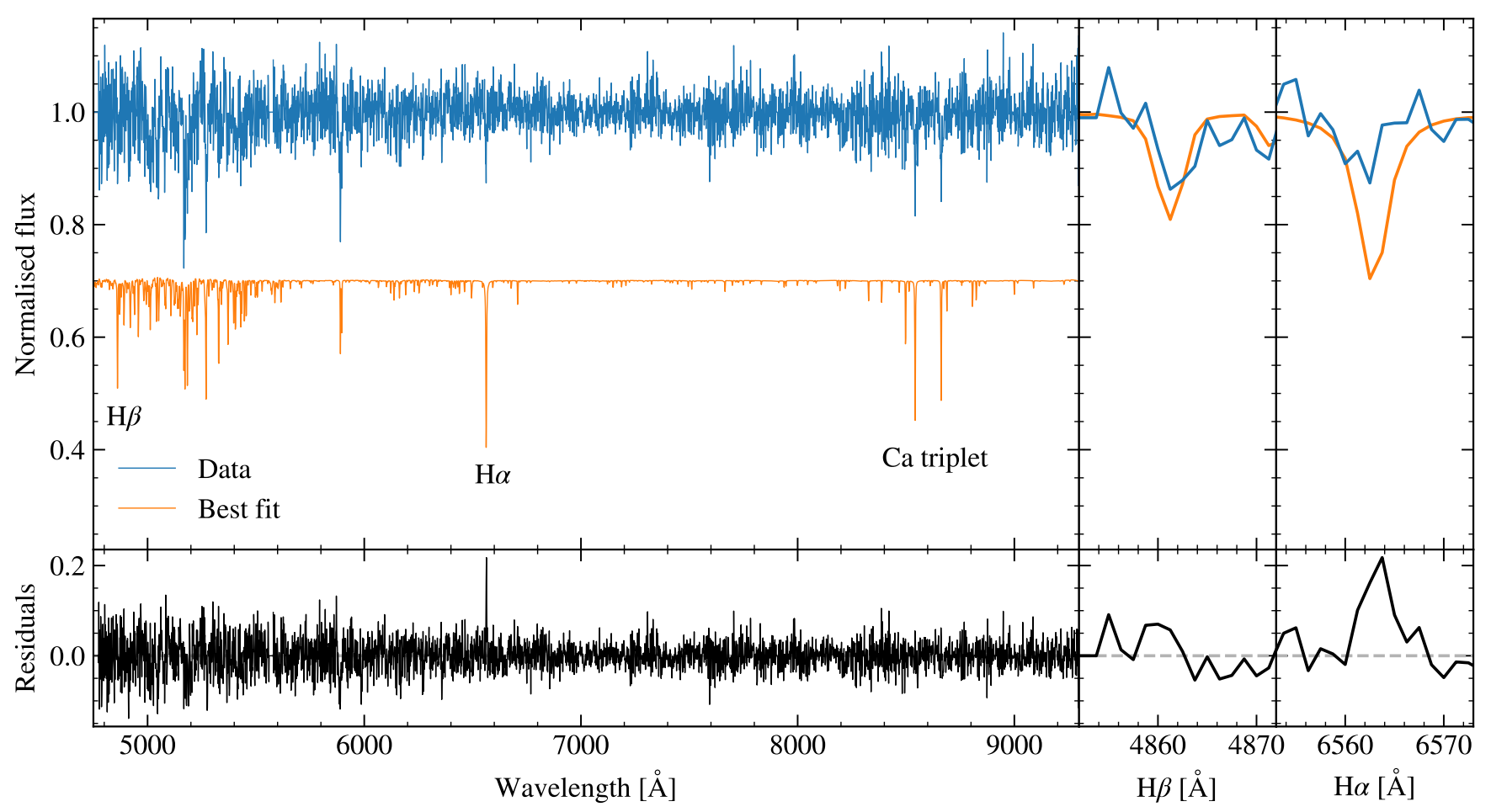

Fig. A.5. Same as Fig. A.3 for the companion of the black hole candidate with ACS Id \#21859.

Table A.1. Radial velocity measurements: 10 randomly selected rows from the electronically published catalogue with $\sim 50000$ rows.

\begin{tabular}{cccccccc}
\hline \hline ACS Id & $\begin{array}{c}\text { RA } \\
\left(^{\circ}\right)\end{array}$ & $\begin{array}{c}\text { Dec } \\
\left(^{\circ}\right)\end{array}$ & $\begin{array}{c}\text { Mag. } \\
\text { F606W }\end{array}$ & $\begin{array}{c}\text { BMJD } \\
(d)\end{array}$ & $\begin{array}{c}v_{\mathrm{r}} \\
\left(\mathrm{km} \mathrm{s}^{-1}\right)\end{array}$ & $\begin{array}{c}\epsilon_{v} \\
\left(\mathrm{~km} \mathrm{~s}^{-1}\right)\end{array}$ & $\begin{array}{c}P\left(\chi_{i}^{2}, v_{i}\right) \\
(\%)\end{array}$ \\
\hline 11317 & 154.41447 & -46.41358 & 15.77 & 2457136.4895 & 491.7 & 1.5 & 100 \\
24879 & 154.38805 & -46.40288 & 19.86 & 2457009.7918 & 499.4 & 6.4 & 36 \\
10605 & 154.42077 & -46.42044 & 18.52 & 2458490.7212 & 481.6 & 3.3 & 17 \\
12088 & 154.40818 & -46.42113 & 19.09 & 2457009.8027 & 491.0 & 4.7 & 4 \\
15271 & 154.38357 & -46.41156 & 20.50 & 2457786.8727 & 496.3 & 7.2 & 16 \\
23145 & 154.40307 & -46.39943 & 20.74 & 2457008.8456 & 490.3 & 7.2 & 60 \\
11426 & 154.41367 & -46.41481 & 18.17 & 2458227.6446 & 506.6 & 8.0 & 35 \\
22284 & 154.41001 & -46.39965 & 20.39 & 2458227.6446 & 517.2 & 8.3 & 13 \\
22723 & 154.40600 & -46.40560 & 18.70 & 2458490.7325 & 478.0 & 4.8 & 7 \\
13914 & 154.39437 & -46.41315 & 21.16 & 2458249.5006 & 495.6 & 8.1 & 7 \\
\hline
\end{tabular}

Notes. ACS Id: identifier in the catalogue of NGC 3201 in the ACS globular cluster survey (Sarajedini et al. 2007). Position and magnitude are also taken from this catalogue. The full table is available at the CDS. 
Table A.2. Binary system properties.

\begin{tabular}{|c|c|c|c|c|c|c|c|c|c|c|}
\hline ACS Id & $\begin{array}{l}\text { RA } \\
\left({ }^{\circ}\right)\end{array}$ & $\begin{array}{l}\text { Dec } \\
\left({ }^{\circ}\right)\end{array}$ & $\begin{array}{l}\text { Mag. } \\
\text { F606W }\end{array}$ & $\begin{array}{r}\text { Peri } \\
\text { (d) }\end{array}$ & & Eccentricity & $\begin{array}{l}\text { Amplitude } \\
\left(\mathrm{km} \mathrm{s}^{-1}\right)\end{array}$ & $\begin{array}{l}\text { Vis. mass } \\
\left(M_{\odot}\right)\end{array}$ & $\begin{array}{l}\text { Invis. min. mass } \\
\qquad\left(M_{\odot} \sin i\right)\end{array}$ & Comment \\
\hline 13108 & 154.40005 & -46.42014 & 17.68 & 0.269 & \pm 0.007 & $0.50 \pm 0.21$ & $33.4 \pm 14.3$ & $1.20 \pm 0.05$ & $0.11 \pm 0.05$ & BSS, $\mathrm{H} \alpha$, EW \\
\hline 25210 & 154.38502 & -46.40803 & 18.05 & 0.3731 & \pm 0.0001 & $0.22 \pm 0.24$ & $9.2 \pm 2.3$ & $0.80 \pm 0.05$ & $0.03 \pm 0.01$ & $\mathrm{H} \alpha$, bimodal \\
\hline 12746 & 154.40330 & -46.41368 & 16.72 & 0.4706 & \pm 0.0001 & $0.01 \pm 0.02$ & $28.0 \pm 0.8$ & $1.20 \pm 0.05$ & $0.12 \pm 0.01$ & BSS, $V \sin i$ \\
\hline 13862 & 154.39460 & -46.42221 & 20.21 & 0.7287 & \pm 0.0001 & $0.09 \pm 0.12$ & $41.9 \pm 6.4$ & $0.60 \pm 0.05$ & $0.14 \pm 0.03$ & $\mathrm{H} \alpha$, bimodal \\
\hline 22697 & 154.40615 & -46.41101 & 19.41 & 0.8631 & \pm 0.0001 & $0.12 \pm 0.10$ & $30.7 \pm 2.4$ & $0.69 \pm 0.05$ & $0.12 \pm 0.02$ & bimodal \\
\hline 12581 & 154.40447 & -46.41137 & 19.09 & 0.89 & \pm 0.43 & $0.14 \pm 0.18$ & $27.1 \pm 3.3$ & $0.72 \pm 0.05$ & $0.10 \pm 0.02$ & bimodal \\
\hline 11222 & 154.41516 & -46.42245 & 16.82 & 0.9430 & \pm 0.0001 & $0.04 \pm 0.04$ & $37.4 \pm 1.8$ & $1.20 \pm 0.05$ & $0.22 \pm 0.02$ & $\mathrm{BSS}, V \sin i$ \\
\hline 13362 & 154.39838 & -46.41245 & 20.86 & 0.9954 & \pm 0.0001 & $0.05 \pm 0.03$ & $124.8 \pm 4.0$ & $0.57 \pm 0.05$ & $0.68 \pm 0.06$ & $\mathrm{H} \alpha$ \\
\hline 12229 & 154.40736 & -46.41683 & 19.83 & 1.6900 & \pm 0.0003 & $0.10 \pm 0.14$ & $15.0 \pm 2.9$ & $0.66 \pm 0.05$ & $0.07 \pm 0.02$ & bimodal \\
\hline 10293 & 154.42364 & -46.41268 & 18.05 & 1.8760 & \pm 0.0001 & $0.04 \pm 0.03$ & $66.3 \pm 1.7$ & $0.80 \pm 0.05$ & $0.44 \pm 0.03$ & \\
\hline 13968 & 154.39393 & -46.41370 & 19.29 & 1.9429 & \pm 0.0001 & $0.05 \pm 0.06$ & $80.9 \pm 7.6$ & $0.71 \pm 0.05$ & $0.55 \pm 0.08$ & $\mathrm{H} \alpha$ \\
\hline 22818 & 154.40547 & -46.39993 & 18.25 & 1.9477 & \pm 0.0002 & $0.24 \pm 0.24$ & $14.7 \pm 5.7$ & $0.79 \pm 0.05$ & $0.08 \pm 0.03$ & bimodal \\
\hline 11802 & 154.41090 & -46.41574 & 19.70 & 2.11 & \pm 0.20 & $0.10 \pm 0.14$ & $20.0 \pm 2.7$ & $0.64 \pm 0.05$ & $0.10 \pm 0.02$ & $\mathrm{H} \alpha$ \\
\hline 21859 & 154.41347 & -46.40691 & 20.37 & 2.2422 & \pm 0.0001 & $0.07 \pm 0.04$ & $305.5 \pm 7.7$ & $0.61 \pm 0.05$ & $7.68 \pm 0.50$ & $\mathrm{BHC}, \mathrm{H} \alpha$ \\
\hline 12836 & 154.40215 & -46.42134 & 20.13 & 2.4262 & \pm 0.0002 & $0.08 \pm 0.08$ & $44.3 \pm 2.5$ & $0.63 \pm 0.05$ & $0.26 \pm 0.03$ & $\mathrm{H} \alpha$ \\
\hline 22692 & 154.40662 & -46.39785 & 17.25 & 5.1038 & \pm 0.0004 & $0.02 \pm 0.03$ & $38.6 \pm 1.6$ & $0.82 \pm 0.05$ & $0.35 \pm 0.03$ & SSG, $\mathrm{H} \alpha+$, X-ray \\
\hline 13438 & 154.39829 & -46.41403 & 17.17 & 5.9348 & \pm 0.0003 & $0.02 \pm 0.03$ & $36.6 \pm 0.9$ & $0.82 \pm 0.05$ & $0.35 \pm 0.03$ & SSG, H $\alpha$, X-ray \\
\hline 15138 & 154.38440 & -46.41622 & 18.14 & 6.393 & \pm 0.002 & $0.05 \pm 0.09$ & $18.8 \pm 2.3$ & $0.79 \pm 0.05$ & $0.16 \pm 0.03$ & bimodal \\
\hline 11366 & 154.41408 & -46.41498 & 19.08 & 8.381 & \pm 0.002 & $0.26 \pm 0.09$ & $43.5 \pm 4.3$ & $0.72 \pm 0.05$ & $0.44 \pm 0.06$ & \\
\hline 23220 & 154.40234 & -46.41006 & 17.05 & 8.428 & \pm 0.002 & $0.08 \pm 0.07$ & $12.9 \pm 1.0$ & $1.20 \pm 0.05$ & $0.15 \pm 0.02$ & BSS \\
\hline 25132 & 154.38563 & -46.41084 & 18.28 & 8.457 & \pm 0.002 & $0.07 \pm 0.08$ & $33.4 \pm 1.9$ & $0.78 \pm 0.05$ & $0.34 \pm 0.04$ & \\
\hline 10799 & 154.41927 & -46.41501 & 18.09 & 8.999 & \pm 0.006 & $0.09 \pm 0.17$ & $9.1 \pm 2.0$ & $0.79 \pm 0.05$ & $0.08 \pm 0.02$ & bimodal \\
\hline 15222 & 154.38393 & -46.41161 & 18.77 & 9.963 & \pm 0.002 & $0.26 \pm 0.09$ & $22.8 \pm 1.9$ & $0.75 \pm 0.05$ & $0.22 \pm 0.03$ & \\
\hline 14749 & 154.38811 & -46.41873 & 17.03 & 10.006 & \pm 0.002 & $0.09 \pm 0.07$ & $42.9 \pm 1.5$ & $0.82 \pm 0.05$ & $0.53 \pm 0.04$ & $\mathrm{SSG}, \mathrm{H} \alpha, \mathrm{EA}$ \\
\hline 10719 & 154.41981 & -46.42151 & 18.99 & 10.930 & \pm 0.004 & $0.03 \pm 0.05$ & $43.7 \pm 2.4$ & $0.74 \pm 0.05$ & $0.54 \pm 0.05$ & \\
\hline 12305 & 154.40665 & -46.41503 & 18.48 & 15.41 & \pm 0.02 & $0.46 \pm 0.14$ & $23.7 \pm 8.9$ & $0.77 \pm 0.05$ & $0.25 \pm 0.09$ & \\
\hline 23918 & 154.39586 & -46.40850 & 18.19 & 15.945 & \pm 0.007 & $0.04 \pm 0.07$ & $32.2 \pm 1.9$ & $0.79 \pm 0.05$ & $0.43 \pm 0.04$ & \\
\hline 10418 & 154.42208 & -46.42361 & 19.07 & 16.128 & \pm 0.005 & $0.11 \pm 0.11$ & $28.5 \pm 2.5$ & $0.72 \pm 0.05$ & $0.35 \pm 0.05$ & \\
\hline 13684 & 154.39605 & -46.42072 & 20.00 & 16.73 & \pm 0.02 & $0.26 \pm 0.14$ & $23.6 \pm 3.4$ & $0.64 \pm 0.05$ & $0.25 \pm 0.05$ & bimodal \\
\hline 11405 & 154.41357 & -46.41997 & 17.25 & 17.219 & \pm 0.006 & $0.42 \pm 0.08$ & $14.1 \pm 1.4$ & $0.82 \pm 0.05$ & $0.15 \pm 0.02$ & SSG, $\mathrm{H} \alpha$ \\
\hline 10274 & 154.42387 & -46.41778 & 16.98 & 18.942 & \pm 0.006 & $0.32 \pm 0.05$ & $24.4 \pm 1.3$ & $1.20 \pm 0.05$ & $0.40 \pm 0.03$ & BSS \\
\hline 12370 & 154.40612 & -46.41486 & 19.46 & 19.46 & \pm 0.02 & $0.16 \pm 0.12$ & $21.8 \pm 2.7$ & $0.70 \pm 0.05$ & $0.26 \pm 0.05$ & \\
\hline 23019 & 154.40364 & -46.40831 & 18.70 & 25.76 & \pm 0.04 & $0.15 \pm 0.15$ & $13.3 \pm 1.9$ & $0.75 \pm 0.05$ & $0.17 \pm 0.03$ & \\
\hline 23847 & 154.39665 & -46.40951 & 16.12 & 26.70 & \pm 0.03 & $0.13 \pm 0.17$ & $6.1 \pm 0.9$ & $0.83 \pm 0.05$ & $0.08 \pm 0.02$ & bimodal \\
\hline 14837 & 154.38719 & -46.42433 & 19.12 & 32.37 & \pm 0.05 & $0.43 \pm 0.18$ & $16.0 \pm 2.0$ & $0.71 \pm 0.05$ & $0.20 \pm 0.04$ & \\
\hline 12706 & 154.40336 & -46.42295 & 18.05 & 34.11 & \pm 0.04 & $0.27 \pm 0.10$ & $12.8 \pm 1.3$ & $0.80 \pm 0.05$ & $0.18 \pm 0.03$ & \\
\hline 12871 & 154.40234 & -46.41310 & 20.35 & 38.2 & \pm 0.2 & $0.09 \pm 0.14$ & $18.3 \pm 2.7$ & $0.61 \pm 0.05$ & $0.27 \pm 0.11$ & bimodal \\
\hline 12341 & 154.40620 & -46.42160 & 17.18 & 38.92 & \pm 0.02 & $0.02 \pm 0.04$ & $22.6 \pm 0.7$ & $0.82 \pm 0.05$ & $0.41 \pm 0.03$ & \\
\hline 21659 & 154.41506 & -46.41032 & 17.29 & 39.52 & \pm 0.02 & $0.49 \pm 0.04$ & $27.7 \pm 2.2$ & $0.82 \pm 0.05$ & $0.45 \pm 0.05$ & \\
\hline 13357 & 154.39872 & -46.41345 & 18.08 & 42.16 & \pm 0.03 & $0.24 \pm 0.07$ & $23.1 \pm 1.2$ & $0.79 \pm 0.05$ & $0.42 \pm 0.04$ & \\
\hline 12308 & 154.40673 & -46.41448 & 17.67 & 47.39 & \pm 0.06 & $0.07 \pm 0.11$ & $29.4 \pm 2.5$ & $0.81 \pm 0.05$ & $0.64 \pm 0.09$ & \\
\hline 12082 & 154.40831 & -46.42402 & 18.09 & 47.88 & \pm 0.09 & $0.26 \pm 0.17$ & $13.0 \pm 2.1$ & $0.79 \pm 0.05$ & $0.21 \pm 0.05$ & \\
\hline 22700 & 154.40637 & -46.41026 & 18.38 & 53.5 & \pm 0.2 & $0.15 \pm 0.18$ & $10.6 \pm 1.7$ & $0.77 \pm 0.05$ & $0.18 \pm 0.04$ & \\
\hline 13221 & 154.39961 & -46.41524 & 18.49 & 53.7 & \pm 0.3 & $0.12 \pm 0.19$ & $13.3 \pm 2.3$ & $0.77 \pm 0.05$ & $0.23 \pm 0.05$ & \\
\hline 13669 & 154.39623 & -46.42264 & 17.45 & 53.8 & \pm 0.1 & $0.13 \pm 0.15$ & $11.7 \pm 1.9$ & $0.82 \pm 0.05$ & $0.21 \pm 0.04$ & \\
\hline 24738 & 154.38877 & -46.41100 & 18.02 & 55.44 & \pm 0.09 & $0.19 \pm 0.10$ & $15.5 \pm 1.7$ & $0.80 \pm 0.05$ & $0.29 \pm 0.04$ & \\
\hline 25266 & 154.38429 & -46.40955 & 18.06 & 55.5 & \pm 0.1 & $0.07 \pm 0.11$ & $13.7 \pm 2.0$ & $0.79 \pm 0.05$ & $0.25 \pm 0.05$ & \\
\hline 13002 & 154.40101 & -46.41437 & 18.29 & 56.4 & \pm 0.2 & $0.53 \pm 0.20$ & $7.1 \pm 1.9$ & $0.78 \pm 0.05$ & $0.10 \pm 0.03$ & bimodal \\
\hline 14889 & 154.38656 & -46.42310 & 18.67 & 56.71 & \pm 0.06 & $0.39 \pm 0.15$ & $27.8 \pm 8.5$ & $0.76 \pm 0.05$ & $0.56 \pm 0.19$ & \\
\hline 14972 & 154.38583 & -46.41625 & 18.02 & 69.2 & \pm 0.2 & $0.18 \pm 0.13$ & $12.2 \pm 1.7$ & $0.80 \pm 0.05$ & $0.23 \pm 0.05$ & \\
\hline 13756 & 154.39561 & -46.41871 & 18.52 & 71.3 & \pm 0.3 & $0.72 \pm 0.16$ & $32.3 \pm 20.0$ & $0.77 \pm 0.05$ & $0.53 \pm 0.33$ & \\
\hline 14743 & 154.38785 & -46.41935 & 18.20 & 74.3 & \pm 0.4 & $0.36 \pm 0.12$ & $20.5 \pm 2.4$ & $0.79 \pm 0.05$ & $0.43 \pm 0.08$ & \\
\hline 23000 & 154.40401 & -46.39918 & 17.61 & 75.3 & \pm 0.3 & $0.20 \pm 0.21$ & $6.3 \pm 1.2$ & $0.81 \pm 0.05$ & $0.12 \pm 0.03$ & \\
\hline 13522 & 154.39741 & -46.41123 & 18.09 & 108.2 & \pm 0.5 & $0.28 \pm 0.09$ & $17.7 \pm 2.3$ & $0.80 \pm 0.05$ & $0.44 \pm 0.08$ & \\
\hline 12904 & 154.40185 & -46.41983 & 16.07 & 110.3 & \pm 0.3 & $0.04 \pm 0.06$ & $11.6 \pm 0.7$ & $1.20 \pm 0.05$ & $0.35 \pm 0.03$ & $\mathrm{BSS}, V \sin i$ \\
\hline 14700 & 154.38856 & -46.41702 & 17.71 & 112.3 & \pm 0.4 & $0.18 \pm 0.18$ & $11.3 \pm 2.0$ & $0.81 \pm 0.05$ & $0.26 \pm 0.06$ & bimodal \\
\hline 13355 & 154.39867 & -46.41374 & 19.93 & 115.1 & \pm 0.6 & $0.63 \pm 0.12$ & $34.9 \pm 10.0$ & $0.67 \pm 0.05$ & $0.82 \pm 0.22$ & \\
\hline 6324 & 154.38867 & -46.42641 & 15.64 & 120.5 & \pm 0.3 & $0.57 \pm 0.12$ & $14.2 \pm 4.8$ & $0.83 \pm 0.05$ & $0.29 \pm 0.08$ & \\
\hline 23175 & 154.40264 & -46.40494 & 15.07 & 129 & \pm 2 & $0.12 \pm 0.20$ & $5.2 \pm 1.4$ & $0.83 \pm 0.05$ & $0.12 \pm 0.04$ & \\
\hline 12927 & 154.40180 & -46.41472 & 19.07 & 143.4 & \pm 0.6 & $0.37 \pm 0.09$ & $14.1 \pm 1.5$ & $0.72 \pm 0.05$ & $0.33 \pm 0.05$ & \\
\hline 11300 & 154.41459 & -46.41824 & 17.00 & 163 & \pm 2 & $0.41 \pm 0.12$ & $17.3 \pm 4.4$ & $0.82 \pm 0.05$ & $0.48 \pm 0.13$ & \\
\hline
\end{tabular}

Notes. The Keplerian parameters were calculated with THE JOKER. The table is sorted by period. ACS Id: Identifier in the catalogue of NGC 3201 in the ACS globular cluster survey (Sarajedini et al. 2007). BH1: Published black hole in Giesers et al. (2018). BHC: Star with a companion that could be a black hole (see Sect. 7.3). BSS: blue straggler star (see Sect. 7.1). Bimodal: The posterior sampling of the periods found by THE JOKER shows two modes. EA: Detached eclipsing binary (Clement 2017). EW: Contact eclipsing binary (Kaluzny et al. 2016). H $\alpha$ : Compared to our best fitting model spectrum (and to a well fitted $\mathrm{H} \beta$ line) several spectra of this star show a partially filled-in $\mathrm{H} \alpha$ absorption line (absorption is not as deep as expected). $\mathrm{H} \alpha+$ : Star showing real $\mathrm{H} \alpha$ emission in several MUSE spectra. NSC: Star with a companion that could be a neutron star. SSG: Sub-subgiant star (see Sect. 7.2). $V$ sin $i$ : star with measured spin velocity according to Simunovic \& Puzia (2014) (see Sect. 7.1). X-ray: Star listed as X-ray source in the November 2017 pre-release of the Chandra Source Catalog Release 2.0 (Evans et al. 2010). 
A\&A 632, A3 (2019)

Table A.2. continued.

\begin{tabular}{|c|c|c|c|c|c|c|c|c|c|c|}
\hline ACS Id & $\begin{array}{l}\text { RA } \\
\left({ }^{\circ}\right)\end{array}$ & $\begin{array}{l}\text { Dec } \\
\left({ }^{\circ}\right)\end{array}$ & $\begin{array}{l}\text { Mag. } \\
\text { F606W }\end{array}$ & & & Eccentricity & $\begin{array}{l}\text { Amplitude } \\
\left(\mathrm{km} \mathrm{s}^{-1}\right)\end{array}$ & $\begin{array}{l}\text { Vis. mass } \\
\quad\left(M_{\odot}\right)\end{array}$ & $\begin{array}{l}\text { Invis. min. mass } \\
\qquad\left(M_{\odot} \sin i\right)\end{array}$ & Comment \\
\hline 12560 & 154.40454 & -46.41537 & 17.57 & 167.01 & \pm 0.09 & $0.61 \pm 0.02$ & $72.3 \pm 1.7$ & $0.81 \pm 0.05$ & $4.53 \pm 0.21$ & BH1 \\
\hline 4513 & 154.40662 & -46.42597 & 17.68 & 172 & \pm 2 & $0.07 \pm 0.12$ & $11.4 \pm 1.6$ & $0.81 \pm 0.05$ & $0.32 \pm 0.06$ & \\
\hline 22751 & 154.40602 & -46.40024 & 14.35 & 172.6 & \pm 0.6 & $0.03 \pm 0.04$ & $10.5 \pm 0.7$ & $0.83 \pm 0.05$ & $0.30 \pm 0.03$ & \\
\hline 12658 & 154.40360 & -46.42207 & 15.40 & 176.0 & \pm 0.4 & $0.07 \pm 0.05$ & $13.4 \pm 0.7$ & $0.83 \pm 0.05$ & $0.40 \pm 0.04$ & \\
\hline 14355 & 154.39107 & -46.41961 & 17.15 & 191 & \pm 3 & $0.06 \pm 0.15$ & $11.1 \pm 1.5$ & $1.20 \pm 0.05$ & $0.41 \pm 0.07$ & BSS, bimodal \\
\hline 12438 & 154.40560 & -46.41283 & 17.22 & 197 & \pm 2 & $0.06 \pm 0.10$ & $8.2 \pm 1.5$ & $0.82 \pm 0.05$ & $0.23 \pm 0.05$ & \\
\hline 23276 & 154.40164 & -46.40921 & 17.51 & 204.2 & \pm 1.0 & $0.89 \pm 0.06$ & $21.2 \pm 10.6$ & $0.82 \pm 0.05$ & $0.30 \pm 0.12$ & \\
\hline 23452 & 154.40018 & -46.40326 & 16.79 & 206 & \pm 2 & $0.06 \pm 0.08$ & $7.2 \pm 0.9$ & $0.82 \pm 0.05$ & $0.20 \pm 0.03$ & \\
\hline 12975 & 154.40134 & -46.42046 & 18.67 & 224 & \pm 4 & $0.07 \pm 0.11$ & $11.5 \pm 1.5$ & $0.77 \pm 0.05$ & $0.35 \pm 0.06$ & \\
\hline 13391 & 154.39790 & -46.42029 & 18.71 & 225 & \pm 6 & $0.24 \pm 0.25$ & $9.8 \pm 2.2$ & $0.76 \pm 0.05$ & $0.28 \pm 0.08$ & \\
\hline 23381 & 154.40051 & -46.40725 & 17.24 & 262 & \pm 2 & $0.11 \pm 0.15$ & $12.1 \pm 1.7$ & $0.82 \pm 0.05$ & $0.42 \pm 0.07$ & \\
\hline 23889 & 154.39636 & -46.40059 & 17.64 & 282 & \pm 5 & $0.33 \pm 0.14$ & $13.2 \pm 2.0$ & $0.81 \pm 0.05$ & $0.43 \pm 0.09$ & \\
\hline 11945 & 154.40948 & -46.41194 & 17.44 & 284 & \pm 8 & $0.18 \pm 0.20$ & $9.8 \pm 2.7$ & $1.20 \pm 0.05$ & $0.40 \pm 0.15$ & BSS \\
\hline 14055 & 154.39350 & -46.41191 & 17.27 & 286 & \pm 5 & $0.17 \pm 0.18$ & $9.0 \pm 1.6$ & $0.82 \pm 0.05$ & $0.29 \pm 0.06$ & \\
\hline 13816 & 154.39521 & -46.41600 & 16.11 & 302 & \pm 5 & $0.17 \pm 0.20$ & $3.9 \pm 1.0$ & $0.83 \pm 0.05$ & $0.11 \pm 0.03$ & \\
\hline 23848 & 154.39645 & -46.40946 & 16.52 & 323 & \pm 2 & $0.48 \pm 0.08$ & $15.6 \pm 1.5$ & $1.20 \pm 0.05$ & $0.67 \pm 0.11$ & BSS \\
\hline 12173 & 154.40785 & -46.41662 & 17.77 & 340 & \pm 6 & $0.47 \pm 0.24$ & $20.0 \pm 7.9$ & $0.81 \pm 0.05$ & $0.80 \pm 0.30$ & bimodal \\
\hline 13069 & 154.40069 & -46.41497 & 17.01 & 375 & \pm 3 & $0.14 \pm 0.19$ & $9.3 \pm 2.7$ & $0.82 \pm 0.05$ & $0.35 \pm 0.14$ & \\
\hline 12932 & 154.40168 & -46.41370 & 17.81 & 386 & \pm 9 & $0.48 \pm 0.20$ & $6.5 \pm 1.9$ & $0.81 \pm 0.05$ & $0.19 \pm 0.07$ & \\
\hline 11831 & 154.41014 & -46.42258 & 13.88 & 493 & \pm 11 & $0.07 \pm 0.14$ & $6.4 \pm 0.9$ & $0.83 \pm 0.05$ & $0.25 \pm 0.04$ & \\
\hline 24135 & 154.39382 & -46.41077 & 18.12 & 509 & \pm 20 & $0.51 \pm 0.30$ & $8.2 \pm 6.7$ & $0.79 \pm 0.05$ & $0.28 \pm 0.18$ & \\
\hline 13587 & 154.39637 & -46.42422 & 18.52 & 537 & \pm 21 & $0.13 \pm 0.15$ & $10.6 \pm 1.8$ & $1.20 \pm 0.05$ & $0.59 \pm 0.14$ & BSS \\
\hline 11317 & 154.41447 & -46.41358 & 15.77 & 602 & \pm 12 & $0.25 \pm 0.13$ & $9.4 \pm 1.4$ & $0.83 \pm 0.05$ & $0.42 \pm 0.07$ & \\
\hline 13874 & 154.39476 & -46.42003 & 18.59 & 608 & \pm 27 & $0.30 \pm 0.30$ & $10.1 \pm 4.0$ & $0.77 \pm 0.05$ & $0.43 \pm 0.20$ & \\
\hline 11779 & 154.41062 & -46.42064 & 17.43 & 620 & \pm 35 & $0.13 \pm 0.21$ & $8.2 \pm 1.7$ & $0.82 \pm 0.05$ & $0.36 \pm 0.10$ & \\
\hline 15293 & 154.38311 & -46.42006 & 16.75 & 668 & \pm 20 & $0.48 \pm 0.10$ & $11.3 \pm 1.8$ & $0.82 \pm 0.05$ & $0.49 \pm 0.10$ & \\
\hline 5132 & 154.40034 & -46.42585 & 20.17 & 764 & \pm 11 & $0.28 \pm 0.16$ & $36.3 \pm 8.4$ & $0.64 \pm 0.05$ & $4.40 \pm 2.82$ & BHC, bimodal \\
\hline 13782 & 154.39552 & -46.41252 & 17.26 & 846 & \pm 61 & $0.11 \pm 0.20$ & $8.2 \pm 1.9$ & $0.82 \pm 0.05$ & $0.42 \pm 0.12$ & \\
\hline 25211 & 154.38487 & -46.40768 & 17.41 & 988 & \pm 75 & $0.11 \pm 0.16$ & $8.7 \pm 1.7$ & $1.20 \pm 0.05$ & $0.59 \pm 0.14$ & BSS \\
\hline 12828 & 154.40192 & -46.42344 & 18.09 & 1075 & \pm 37 & $0.05 \pm 0.08$ & $13.2 \pm 1.4$ & $0.80 \pm 0.05$ & $0.90 \pm 0.16$ & \\
\hline 11131 & 154.41636 & -46.41623 & 17.28 & 1627 & \pm 222 & $0.31 \pm 0.17$ & $8.2 \pm 1.6$ & $0.82 \pm 0.05$ & $0.54 \pm 0.13$ & bimodal \\
\hline 23215 & 154.40267 & -46.39830 & 18.73 & 1997 & \pm 304 & $0.08 \pm 0.14$ & $16.3 \pm 2.1$ & $0.76 \pm 0.05$ & $1.82 \pm 0.39$ & NSC \\
\hline 13154 & 154.39996 & -46.41225 & 18.17 & 2773 & \pm 1009 & $0.16 \pm 0.19$ & $10.1 \pm 4.1$ & $0.79 \pm 0.05$ & $0.94 \pm 0.94$ & \\
\hline 13808 & 154.39505 & -46.41923 & 15.23 & 3001 & \pm 1005 & $0.11 \pm 0.22$ & $3.2 \pm 1.4$ & $0.83 \pm 0.05$ & $0.21 \pm 0.13$ & \\
\hline
\end{tabular}

